\title{
The form and orientation of Langmuir cells for misaligned winds and waves
}

\author{
L. P. Van Roekel, ${ }^{1}$ B. Fox-Kemper, ${ }^{1,2}$ P. P. Sullivan, ${ }^{3}$ P. E. Hamlington, ${ }^{4}$ and S. R. Haney ${ }^{1,2}$
}

Received 15 August 2011; revised 29 February 2012; accepted 5 March 2012; published 1 May 2012.

[1] Large eddy simulations of the Craik-Leibovich equations are used to assess the effect of misaligned Stokes drift and wind direction on Langmuir cells in the ocean mixed layer. Misalignments from $0^{\circ}$ to $135^{\circ}$ are examined and Langmuir turbulence structures are evident in all cases. The Stokes drift is modeled using a broadband empirical spectrum, and cases with and without the Coriolis effect, wind waves, and an initial mixed layer are examined. The expected scaling for the vertical velocity variance is recovered in the aligned simulations and is adapted here to the misaligned cases. The adjusted scaling projects the friction velocity (aligned with the wind stress) into the dominant axial direction of the Langmuir cells. The turbulent Langmuir number is generalized through a similar projection into the axial direction of the Langmuir cells, which reduces its value in realistic conditions. For known Langmuir cell orientations, the strength of Langmuir turbulence for misaligned cases can be estimated using the projected Langmuir number. A prediction for the angle between the wind stress and cell direction is obtained using the law of the wall; this prediction only requires the wind stress, Stokes drift, and boundary layer depth.

Conditional analyses show that, with increasing misalignment, the typically antisymmetric Langmuir cell pairs become asymmetric. This asymmetry is due, in part, to the advection by cross cell flow of vorticity from one vortex tube onto the other, and in part due to an asymmetry induced by the stretching of vertical vorticity into cross cell vorticity.

Citation: Van Roekel, L. P., B. Fox-Kemper, P. P. Sullivan, P. E. Hamlington, and S. R. Haney (2012), The form and orientation of Langmuir cells for misaligned winds and waves, J. Geophys. Res., 117, C05001, doi:10.1029/2011JC007516.

\section{Introduction}

[2] The ocean surface layer is the intermediary for oceanatmosphere fluxes of momentum and tracers. Small scale phenomena are critical to the creation and maintenance of this layer. Processes at submesoscale lengths and below can have a dramatic impact on global climate [Large et al., 1997; Fox-Kemper et al., 2011; A. Webb et al., Global climate model sensitivity to estimated Langmuir mixing, manuscript in preparation, 2011], and the surface wavefield impacts the upper ocean directly through the breaking of waves and the corresponding transfer of momentum into the surface layer [Craig and Banner, 1994; Sullivan and McWilliams, 2010; Cavaleri et al., 2012].

[3] Even surface waves that do not break can impact the upper ocean. The Stokes drift associated with surface waves

\footnotetext{
${ }^{1}$ Cooperative Institute for Research in Environmental Sciences, University of Colorado Boulder, Boulder, Colorado, USA.

${ }^{2}$ Department of Atmospheric and Oceanic Sciences, University of Colorado Boulder, Boulder, Colorado, USA.

${ }^{3}$ Mesoscale and Microscale Meteorology Division, National Center for Atmospheric Research, Boulder, Colorado, USA.

${ }^{4}$ Aerospace Engineering Sciences, University of Colorado Boulder, Boulder, Colorado, USA.

Copyright 2012 by the American Geophysical Union. 0148-0227/12/2011JC007516
}

tilts vertical vorticity anomalies into the horizontal. The instability that governs this process was described by Craik [1977], and leads to the creation of vortices called Langmuir Cells (LC) [Langmuir, 1938]. LC are a feature of the waveand wind-driven turbulence of the upper ocean, which here will be called Langmuir Turbulence (LT) [after McWilliams et al., 1997, hereinafter MSM97]. Similar to breaking waves, LT tends to mix the upper ocean, although mixing by LT extends far below the surface, sometimes even deep enough to entrain water across the mixed layer base.

[4] LT has been studied in recent years through field campaigns [Weller and Price, 1988; Smith, 1992; D'Asaro and Dairiki, 1997] and large eddy simulations (LES) of the Craik-Leibovich (CL) equations [Craik, 1977; Skyllingstad and Denbo, 1995; McWilliams et al., 1997; Noh et al., 2004; Sullivan et al., 2004, 2007; Li et al., 2005; Polton and Belcher, 2007; Grant and Belcher, 2009]. The CL equations are obtained by wave averaging the Navier-Stokes equations, and the effect of the Stokes drift velocity, $\mathbf{u}_{s}$, enters through additional forcing of the momentum, turbulent kinetic energy, and Eulerian vorticity budgets. Kukulka et al. [2009, 2010] have shown that results from LES are in good agreement with observations, and LES has provided substantial insights into the governing processes of LT.

[5] Using results from LES, McWilliams and Sullivan [2000] suggest that the ratio of mixing due to LT over that due to ordinary shear turbulence (ST) (i.e., neglecting Stokes 
drift forcing) should scale with the turbulent Langmuir number, $L a_{t}^{2} \equiv u_{*} / u_{s}(0)$. Here, $u_{*} \equiv \sqrt{|\tau| / \rho}$ is the surface friction velocity, $\tau$ is the surface wind stress, $\rho$ is the density, and $u_{s}(0)$ is the surface value of the Stokes drift velocity in the direction of the wind stress. Scalings of the strength of Langmuir mixing (i.e., mixing that is directly associated with LT) have also been proposed in several other studies [Grant and Belcher, 2009; Kukulka et al., 2010], and recent simulations [Sullivan et al., 2004, 2007; Noh et al., 2004] have additionally examined the impact of wave breaking on LT and the upper ocean.

[6] Despite increasing LES complexity, most prior studies have assumed that the Stokes drift is aligned with the surface wind stress (i.e., that $\tau$ and $\mathbf{u}_{s}$ point in the same direction). The case of misaligned wind and waves is, however, important for understanding the dynamics of LC and the impact of LT on the mixed layer in more realistic situations. Hanley et al. [2010] argue that seas are rarely in wind-wave equilibrium, and that the wavefield contains both swell and wind waves (although the implications of this result in terms of wave age are less clear [Högström et al., 2011; Hanley et al., 2011]). Swell waves, in particular, are susceptible to misalignment with the wind direction [Hanley et al., 2010]. Similar scenarios may result from a change of wind (e.g., during a hurricane), waves propagating from one region to another, or wave refraction. Observations from Hurricane Gustav and Typhoon Megi, for example, show vastly different mixing signatures for different wind-wave orientations (E. A. D’Asaro, personal communication, 2011).

[7] The impact of misaligned wind and waves on LC has been studied previously, although not through full LES of the non-linear CL equations. Gnanadesikan and Weller [1995] suggest that LC will align with the direction of maximum Lagrangian velocity shear, where the Lagrangian velocity vector, $\mathbf{u}_{L}$, is defined as the sum of Eulerian, $\mathbf{u}$, and Stokes currents as $\mathbf{u}_{L}=\mathbf{u}+\mathbf{u}_{s}$. This result has also been confirmed by linear stability analysis [Polonichko, 1997; Cox, 1997]. Since these studies, however, the problem has lain dormant, and little progress has been made in understanding LT in the misaligned case using simulations of the non-linear CL equations.

[8] In this paper, LES of the CL equations are used to understand the properties of LT when the wind and waves are misaligned. The simulations use the basic problem formulation outlined by MSM97, but with the wind and wave directions decoupled. In each simulation, the wind stress and Stokes drift are constant in space and time, but the angle between the wind and waves, denoted $\theta_{w w}$, is varied. We consider angles between $0^{\circ}$ and $135^{\circ}$, and find that LC are present for all $\theta_{w w}$, even for $\theta_{w w} \geq 90^{\circ}$. By $135^{\circ}$, the LT characteristics are quite different from the $0^{\circ}$ to $90^{\circ}$ cases, but weakly anisotropic structures resembling LC still exist. Only in the most extreme case (i.e., $\theta_{w w}=180^{\circ}$ ), where the Stokes drift directly opposes the wind stress, is there minimal generation of LT. In this case, the Stokes drift shear nearly cancels the shear induced by the wind. Following Gnanadesikan and Weller [1995], we also examine cases with and without the effects of rotation (Coriolis force).

[9] One of the challenges of examining LC is that in the LT regime, disordered bunches of LC combine with other features associated with ST. Through comparisons with simulations in the absence of Stokes drift, we distinguish LT/LC structures from those that are similar to structures in ordinary ST. Harcourt and D'Asaro [2008] also argue that the wave spectra used to force LES models can have a dramatic impact on the scaling of LT.

[10] Many previous LES studies have used a Stokes drift profile from monochromatic waves, but Harcourt and D'Asaro [2008] have shown that an empirical wave spectrum with explicit dependence on wave age, defined as the phase speed of the peak wave $\left(C_{p}\right)$ normalized by the ten meter wind speed $\left(U_{10}\right)$, should be used for more realistic forcing. Consequently, an empirical spectrum is used here to represent the vertical profile of the Stokes drift velocity. To ensure robust results, additional simulations have been carried out that include superposition of a second set of young waves aligned with the wind, with negligible consequences.

[11] Even though there is sensitivity to the form of the wave spectrum used in the formulation of $\mathbf{u}_{s}$, Harcourt and D'Asaro [2008] show that the bulk (i.e., mixed layer average) value of vertical kinetic energy predicted by monochromatic wave spectra collapses to the result from broadband empirical spectra if $L a_{t}$ is redefined using an average over the surface layer. Harcourt and D'Asaro [2008] take the depth of this layer as roughly $20 \%$ of the mixed layer depth $\left(H_{M L}\right)$. As a result, their generalized formulation for $L a_{t}$ addresses cases with either empirical or monochromatic wave spectra. A similar procedure is used here to formulate an expression for $L a_{t}$ that addresses cases where the effects of the Stokes drift penetrate more deeply into the mixed layer, and where wind and Stokes drift are misaligned. It will be shown that the relative alignment of winds and waves is critically important in determining the strength of mixing by LT. Thus, misalignment should be considered in studies of the distribution of Langmuir number globally [Webb and Fox-Kemper, 2011; S. E. Belcher et al., A global perspective on mixing in the ocean surface boundary layer, unpublished manuscript, 2011].

[12] The primary goals of this study are: (1) to characterize changes in the properties of LC as a function of $\theta_{w w}$, Coriolis force, and Stokes drift magnitude, (2) to briefly explore the differences in energy and vorticity budgets that result from misalignment and their effects on the resulting LC, (3) to find a successful prediction for the orientation of the LC relative to the directions of the wind stress and Stokes drift, and (4) to show that by projecting the wind stress and Stokes drift into the direction of LC, standard LT scalings are nearly recovered. Details of the simulations are provided in section 2, properties of the LC are given in section 3, kinetic energy and vorticity budgets are discussed in section 4 , predictions for the orientations of LC are presented in section 5, conditional analyses of LC are presented in section 6 , and scalings of $L a_{t}$ are addressed in section 8 .

\section{Model and Simulation Description}

[13] The simulations are carried out using the LES model developed at NCAR [Moeng, 1984], which solves the spatially filtered CL equations given by [McWilliams et al., 1997; Holm, 1996]

$$
\frac{\partial \rho}{\partial t}+\mathbf{u}_{L} \cdot \nabla \rho=\mathrm{SGS}
$$


Table 1. Parameters Used in the Simulations ${ }^{\mathrm{a}}$

\begin{tabular}{cccccccc}
\hline$U_{10}(\mathrm{~m} / \mathrm{s})$ & $u_{*}(\mathrm{~m} / \mathrm{s})$ & $\left|\mathbf{u}_{s}(0)\right|(\mathrm{m} / \mathrm{s})$ & $\overline{w^{\prime} \theta^{\prime}}{ }_{s f c}\left(\mathrm{~W} / \mathrm{m}^{2}\right)$ & $f\left(\mathrm{~s}^{-1}\right)$ & $H_{M L}(\mathrm{~m})$ & $n x, n y, n z$ & $d x, d y, d z(\mathrm{~m})$ \\
\hline 5.75 & $5.3 \cdot 10^{-3}$ & $0.06313,+\mathrm{ww}$ & -5 & $0,10^{-4}$ & 0,33 & $128,128,160$ & $2.5,2.5,0.6$ \\
\hline
\end{tabular}

${ }^{a}$ Here $U_{10}$ is the $10 \mathrm{~m}$ simulation wind speed, $u *$ is the surface friction velocity, $\mathbf{u}_{s}(0)$ is the near-surface Stokes drift, which is located at the first grid point at $-0.3 \mathrm{~m}$, (+ww implies that some runs included aligned wind waves), $\overline{w^{\prime} \theta^{\prime}}{ }_{s f c}$ is the cooling applied at the surface, $f$ is the Coriolis parameter, $H_{M L}$ is the initial mixed layer depth, $n x, n y, n z$ are the computational dimensions and $d x, d y, d z$ are the grid cell widths. In the present simulations, $f, H_{M L}$ and $\left|\mathbf{u}_{s}(0)\right|$ are altered while the remaining parameters are held constant.

$$
\begin{gathered}
\nabla \cdot \mathbf{u}=0 \\
\frac{\partial \mathbf{u}}{\partial t}+(\boldsymbol{\omega}+f \hat{\mathbf{z}}) \times \mathbf{u}_{L}=-\nabla \pi-\frac{g \rho \hat{\mathbf{z}}}{\rho_{0}}+\mathrm{SGS},
\end{gathered}
$$

where the Lagrangian velocity is $\mathbf{u}_{L}=\mathbf{u}+\mathbf{u}_{s}$, the Eulerian vorticity is $\boldsymbol{\omega}=\nabla \times \mathbf{u}, f$ is the Coriolis parameter, $g$ is the acceleration due to gravity, $\rho_{0}$ is a reference density, SGS denotes subgrid-scale terms (here, the two-part SGS model of Sullivan et al. [1996]), and $\pi$ is an effective pressure given by

$$
\pi=\left(\frac{p}{\rho_{0}}+\frac{1}{2}\left|\mathbf{u}_{L}\right|^{2}\right)
$$

The equation of state is given as $\rho=\rho_{0}\left(1-\beta_{T} \theta\right)$, where $\theta$ is the temperature and $\beta_{T}$ is the coefficient of thermal expansion. The surface boundary conditions consist of a slight cooling to initiate convection and a wind stress, $\tau$, to which the $x$-axis is aligned.

[14] Forcing by surface waves enters the CL equation (3) through the $\boldsymbol{\omega} \times \mathbf{u}_{s}$ term, the Coriolis term, $f \hat{\mathbf{z}} \times \mathbf{u}_{s}$, and the effective pressure in (4). The Stokes drift also contributes to the advection of density in (1). For misaligned wind and waves, the Stokes drift velocity, $\mathbf{u}_{s}$, can be written in the wind-based coordinates as

$$
\mathbf{u}_{s}(z)=U_{s}(z)\left[\cos \left(\theta_{w w}\right) \hat{\mathbf{x}}+\sin \left(\theta_{w w}\right) \hat{\mathbf{y}}\right]
$$

where $\theta_{w w}$ is the angle between the wind and waves, and the $\hat{\mathbf{x}}$ direction is aligned with the wind. The vertical Stokes drift profile, $U_{s}(z)$, is represented in the present study by a broadband empirical spectrum, as opposed to the monochromatic spectrum used in prior studies [e.g., McWilliams et al., 1997]. Two different empirical spectra for wind and equilibrated waves from Donelan et al. [1985] are considered here: high frequency young waves with a wave age of $C_{p} / U_{10}=0.25$ and waves in a fully developed sea with $C_{p} / U_{10}=1.2$. The resulting $U_{s}(z)$ from the Donelan et al. [1985] spectra decays super-exponentially with distance from the surface [Webb and Fox-Kemper, 2011], and thus we expect LC to be more confined to the surface than in simulations using monochromatic wavefields. Since the Donelan et al. [1985] spectrum does not have a well-defined surface Stokes drift value, throughout we use the Stokes drift at the first grid point, $U_{s}(-0.3 \mathrm{~m})$, as our value for $U_{s}(0)$. This value most accurately represents the resolved Stokes drift shear available for production. In the present simulations, the surface shear velocity can be parameterized through the skin friction velocity, which is given as $u_{*}=5.27 \times 10^{-3} \mathrm{~ms}^{-1}$.

[15] As in previous studies of aligned wind and waves [e.g., McWilliams et al., 1997], some time is required after the initial onset of simulated turbulence before the statistical properties of the turbulence are reliable. The simulations of misaligned waves and wind are not fundamentally different, and so care is taken to analyze the spin-up and stabilization of the results presented. The analysis window begins when spin-up appears complete, but the continuing variations were monitored. Cases where the coordinate system is taken as rotating are particularly prone to difficulty as inertial oscillations persist throughout the simulation. Thus, when a variable is not stable, averages are carried out over part or all of the inertial oscillation cycle.

[16] Simulations have been carried out for wind-wave misalignments $\theta_{w w}=0^{\circ}, 30^{\circ}, 45^{\circ}, 60^{\circ}, 90^{\circ}$, and $135^{\circ}$. These angles have been examined for both rotating $\left(f=10^{-4} \mathrm{~s}^{-1}\right)$ and non-rotating $\left(f=0 \mathrm{~s}^{-1}\right)$ cases. Forcing parameters are the same in all simulations (see Table 1), and the only changes are the values of $\theta_{w w}$ and $f$. Sensitivity to a number of additional parameters has also been examined. In particular, aligned wind waves with $C_{p} / U_{10}=0.25$ are superimposed on misaligned waves with $C_{p} / U_{10}=1.2$ (see Table 1 ), resulting in a perturbed Stokes drift. Simulations have also been carried out without an initial mixed layer, and using a variety of different magnitudes for $U_{s}(z)$ in order to give a range of values for $L a_{t}$ (see Tables 1 and 2).

[17] The baseline simulations are initialized with a mixed layer that extends $33 \mathrm{~m}$ below the surface, with constant stratification below this depth. The velocity field is zero everywhere except at the surface where there is divergencefree noise. The domain size is $320 \mathrm{~m} \times 320 \mathrm{~m} \times 96 \mathrm{~m}$ in the $x, y$, and $z$ directions respectively, with $128 \times 128 \times$ 160 computational cells. Sensitivity to grid resolution was examined by doubling the number of points used in all directions. While quantitative differences $(\leq 20 \%)$ were observed in certain statistics, for example the peak vertical kinetic energy (VKE), the insights and trends obtained in the present study were unchanged. The Stokes drift from the

Table 2. Parameters Used in Simulations Examining Varying Stokes Drift Magnitude ${ }^{\mathrm{a}}$

\begin{tabular}{lccccc}
\hline$\theta_{w w}$ & $\begin{array}{c}\text { Preconditioning } \\
U_{10}(\mathrm{~m} / \mathrm{s})\end{array}$ & $\mathbf{u}_{s}(0)(\mathrm{m} / \mathrm{s})$ & $f_{p}(\mathrm{~Hz})$ & $L a_{t}$ & $k_{p}^{-1}(\mathrm{~m})$ \\
\hline $0,30,60$ & 1.15 & 0.022 & 0.38 & 0.49 & 1.7 \\
$0,30,60$ & 2.875 & 0.038 & 0.28 & 0.37 & 3.2 \\
$0,30,60$ & 5.75 & 0.063 & 0.22 & 0.29 & 5.1 \\
$0,30,60$ & 11.5 & 0.100 & 0.18 & 0.23 & 7.7 \\
$0,30,60$ & 28.75 & 0.162 & 0.13 & 0.18 & 14.7 \\
\hline
\end{tabular}

${ }^{\mathrm{a}}$ Here $\theta_{w w}$ is the wind-wave angle, $U_{10}$ is the preconditioning wind velocity, $\mathbf{u}_{s}(0)$ is the near-surface Stokes drift, $f_{p}$ is the peak wave frequency, and $L a_{t}^{2}=u_{*} /\left|\mathbf{u}_{s}(0)\right|$ is the turbulent Langmuir number. Note that even though the preconditioning winds are varied, the simulation winds are always $U_{10}=5.75 \mathrm{~m} / \mathrm{s}$. Also note that the vertical resolution remains fixed, which limits the amount of Stokes shear present for the largest $f_{p}$. To elucidate, $k_{p}^{-1}$, the $e$-folding depth of the peak wave, is also included. 


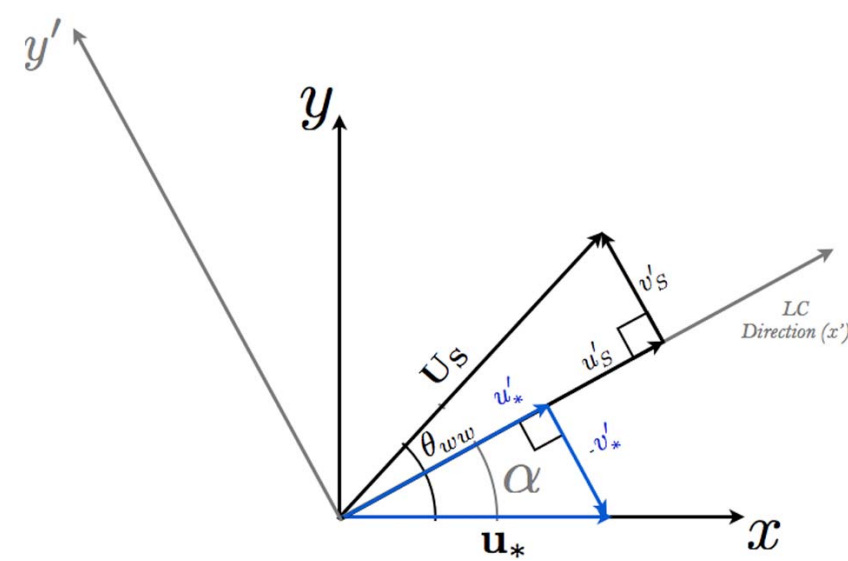

Figure 1. Schematic of coordinate rotation used in this work. The blue arrows represent the wind-forcing and the thin black line is the surface Stokes drift. All of the primed quantities are the surface forcing seen in the rotated framework.

highest-frequency waves are not well-represented, which is why we choose to use the near-surface Stokes drift rather than estimate an unresolved surface value in our scalings. In the rotating simulations with Coriolis force, the model is run long enough for one inertial oscillation to be completed.

[18] In examining the case of misaligned wind and waves, there are a number of different vector quantities to consider (see Figure 1). The three primary directions treated here are (1) the direction of the Stokes drift (labeled $\mathbf{u}_{s}$ in Figure 1), (2) the direction of the wind stress and skin friction velocity (labeled $\mathbf{u}_{*}$ in Figure 1), and (3) the dominant direction of the Langmuir cells (labeled "LC direction" in Figure 1). Herein, $\mathbf{u}_{*}$ is used as a shorthand for $\mathbf{u}_{*}=u * \tau /|\tau|$, but the reader should note that $u *$ is not ordinarily a vector, only a scaling for the vertical velocity shear (which is a vector). Two angles completely describe the relative directions of these three horizontal vectors: $\theta_{w w}$, which is the angle between the wind stress and the Stokes drift, and $\alpha$, which is the direction of the LC axis relative to the wind. The angle between the LC axis and the Stokes drift is therefore $\theta_{w w}-\alpha$. In all simulations, $\mathbf{u}_{*}$ is assumed to be aligned with the $x$-axis.

[19] Allowing the LC direction to differ from the Stokes drift and wind shear directions is crucial; in particular, it is the dynamical balances in the LC direction that are most closely preserved as the wind and wave forcing varies. For this reason, a rotated coordinate system $\left(x^{\prime}\right.$ and $y^{\prime}$ in Figure 1) aligned with the LC will be exploited. Note that it is not true that the Eulerian velocity, $\mathbf{u}$, has the magnitude or direction of $\mathbf{u}_{*}$; for example, in a rotating frame of reference the Coriolis effect deflects the currents in an Ekman spiral. In the following, the direction of the Ekman spiral at each depth will prove to be less predictive than the three vectors shown in Figure 1, consistent with the large Rossby number of the LT.

[20] In many of the simulations, the equilibrium wave direction and magnitude have been separated from the wind stress used during the simulation. To determine a realistic magnitude of the fully developed waves, the Stokes drift is computed by using the magnitude of a 'preconditioning wind stress' (see Table 2). To understand this approach, consider a fully developed sea with an appropriate wavefield $\left(C_{p} / U_{10}=1.2, U_{s}(0)=0.06313 \mathrm{~m} / \mathrm{s}\right)$ matched to a given preconditioning wind stress $\left(U_{10}=5.75 \mathrm{~m} / \mathrm{s}\right.$ in the direction of $\mathbf{u}_{s}$ in Figure 1). After these equilibrium waves are established, suppose that the wind changes in direction and magnitude to align with $\mathbf{u}_{*}$ in Figure 1 . The situation after the wind changes is what motivates the basic simulation herein. Note that the preconditioning wind is only a hypothetical wind used to construct a realistic Stokes drift; it is never used to generate wind stresses during the simulation. The winds after the change in direction and magnitude are the 'simulation' winds. In reality, the simulation winds should also generate developing wind waves superimposed on the older waves, and the older waves should evolve toward a swell spectrum. In most of the simulations described here, the development of aligned wind waves and misaligned swell is ignored. In section 7 , a few simulations add the aligned wind waves by superposition of a younger Donelan wave spectrum $\left(C_{p} / U_{10}=0.25\right)$ aligned with the wind. In section 8 , the effects of varying the preconditioning wind speed are presented. The simulation wind speed is, however, always maintained at $U_{10}=5.75 \mathrm{~m} / \mathrm{s}$.

[21] It is important to note that many effects are neglected in the model used here. In the real world, the evolution of the wavefield would be simultaneous with the development of the turbulence. The modeling framework used here does not allow for a realistic portrayal of the evolution of the wavefield details in response to the changing wind during the simulation. Likewise, realistic coupling of turbulence to a true free surface with the effects of breaking waves and higher-order estimates of the Stokes drift are not considered. Someday, a direct numerical simulation of the Navier-Stokes equations rather than a LES of the CL equations will be possible. However, the conceptual framework and results presented here are a useful starting point against which the effect of these more challenging modeling scenarios can be quantified. For many purposes, such as climate model parameterizations or construction of climatologies where the Stokes drift is highly uncertain in any case [Webb and Fox-Kemper, 2011], these results are likely sufficiently accurate.

\section{Langmuir Cell Properties}

[22] Figure 2 shows fields of the vertical velocity fluctuation, $w^{\prime}$, for $\theta_{w w}=0^{\circ}-135^{\circ}$. The fields are for the nonrotating simulations, and are shown at $z=-1.5 \mathrm{~m}$, which is the depth of maximum VKE, $\overline{w^{\prime 2}}$, for most values of $\theta_{w w}$ examined here. The average $\bar{\varphi}$ (where $\varphi$ is some variable) corresponds to an average over the $x$ and $y$ directions, and is generally a function of both vertical depth, $z$, and time, $t$. In Figure 2, the wind stress is to the right, parallel to the $x$ axis, and the Stokes drift direction is given by $\theta_{w w}$, measured with respect to the $x$-axis. Figure 2 shows that the angle associated with the upwelling (positive $w^{\prime}$ ) and downwelling (negative $w^{\prime}$ ) regions increases with increasing $\theta_{w w}$. These regions are characteristic of LC and correspond to the 'windrows' observed by Langmuir [1938] and many others. For all cases where $\theta_{w w}>0^{\circ}$, Figure 2 shows that the windrows are oriented between the Stokes drift and 


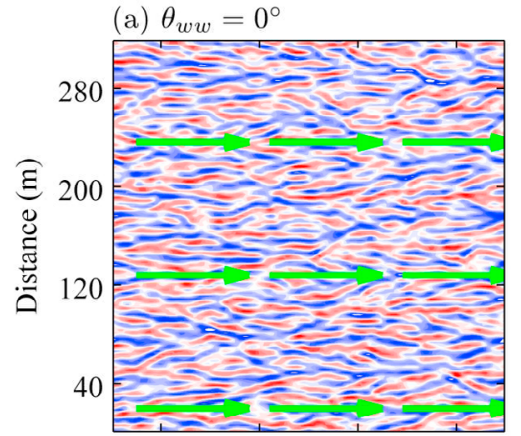

(d) $\theta_{w w}=60^{\circ}$

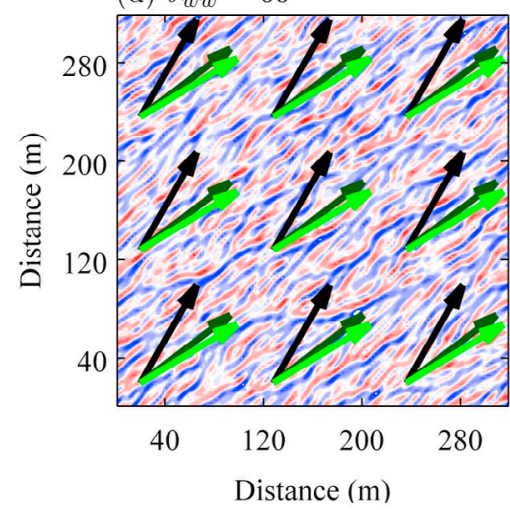

(b) $\theta_{w w}=30^{\circ}$

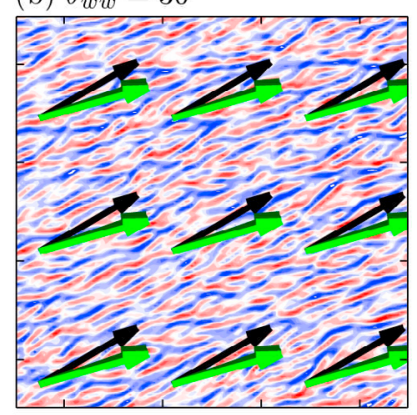

(e) $\theta_{w w}=90^{\circ}$

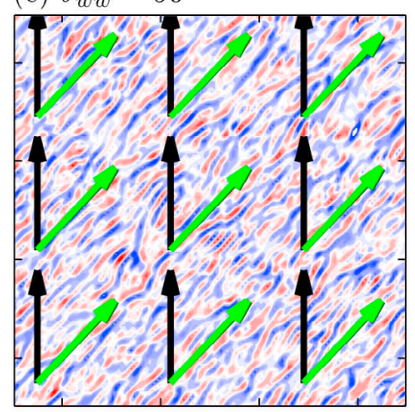

40

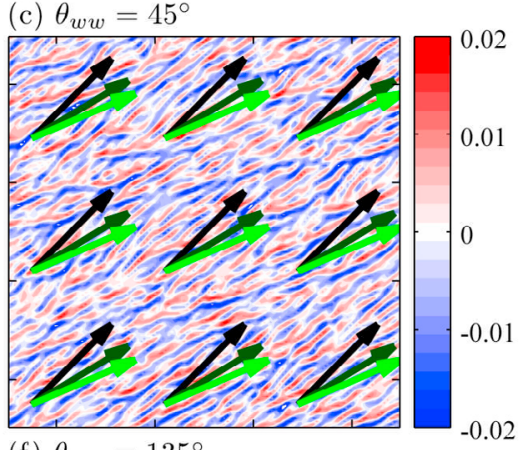

(f) $\theta_{w w}=135^{\circ}$
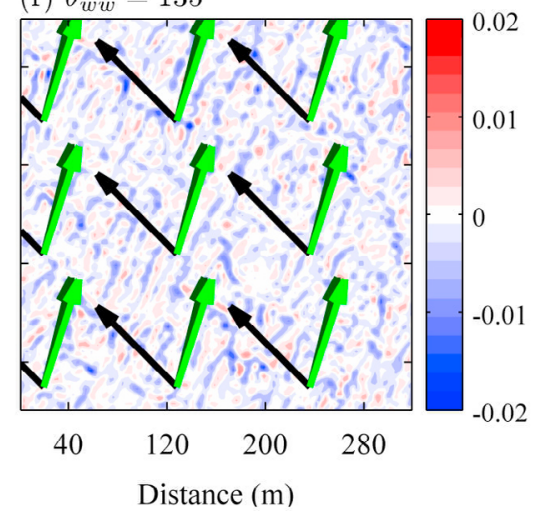

Distance (m)

Distance (m)

Figure 2. (a-f) Contours of vertical velocity, $w^{\prime}$, at the depth of maximum vertical velocity variance $(z=-1.5 \mathrm{~m})$ for non-rotating $\left(f=0 \mathrm{~s}^{-1}\right)$ simulations with $\theta_{w w}=0^{\circ}-135^{\circ}$. The black arrows are the Stokes drift direction (making an angle $\theta_{w w}$ with the $x$-axis), the dark green arrows are the LC direction from Figure 4, with angles $\alpha$ given in Table 3, and the light green arrows are the direction given by the law of the wall angle, $\alpha_{L O W}$, from (18).

wind directions. This suggests that LC are not aligned with the Stokes drift direction when waves and wind are misaligned. Moreover, even though the strength of the upwelling and downwelling regions decreases with increasing $\theta_{w w}$, relatively weak windrows are still present even for $\theta_{w w}=135^{\circ}$.

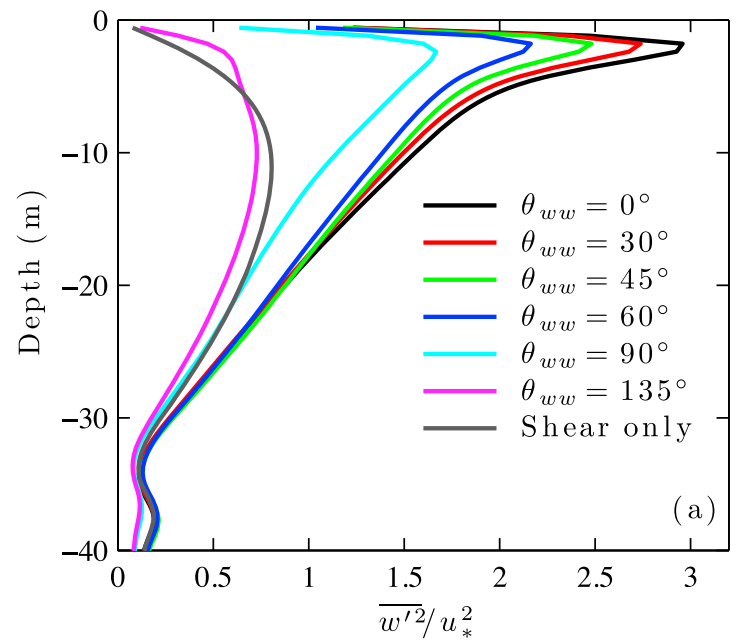

[23] This last result can be confirmed quantitatively by considering the profiles of $\overline{w^{\prime 2}}$ (VKE) shown in Figure 3. For both rotating and non-rotating cases, Figure 3 shows that $\overline{w^{\prime 2}}$ peaks near the surface and decreases with increasing depth. Although there are only small differences between the

Figure 3. Vertical profiles of $\overline{w^{\prime 2}}$ normalized by $u_{*}^{2}$ for (a) non-rotating and (b) rotating cases. Results are shown for $\theta_{w w}=0^{\circ}-135^{\circ}$ and for the shear only case. 

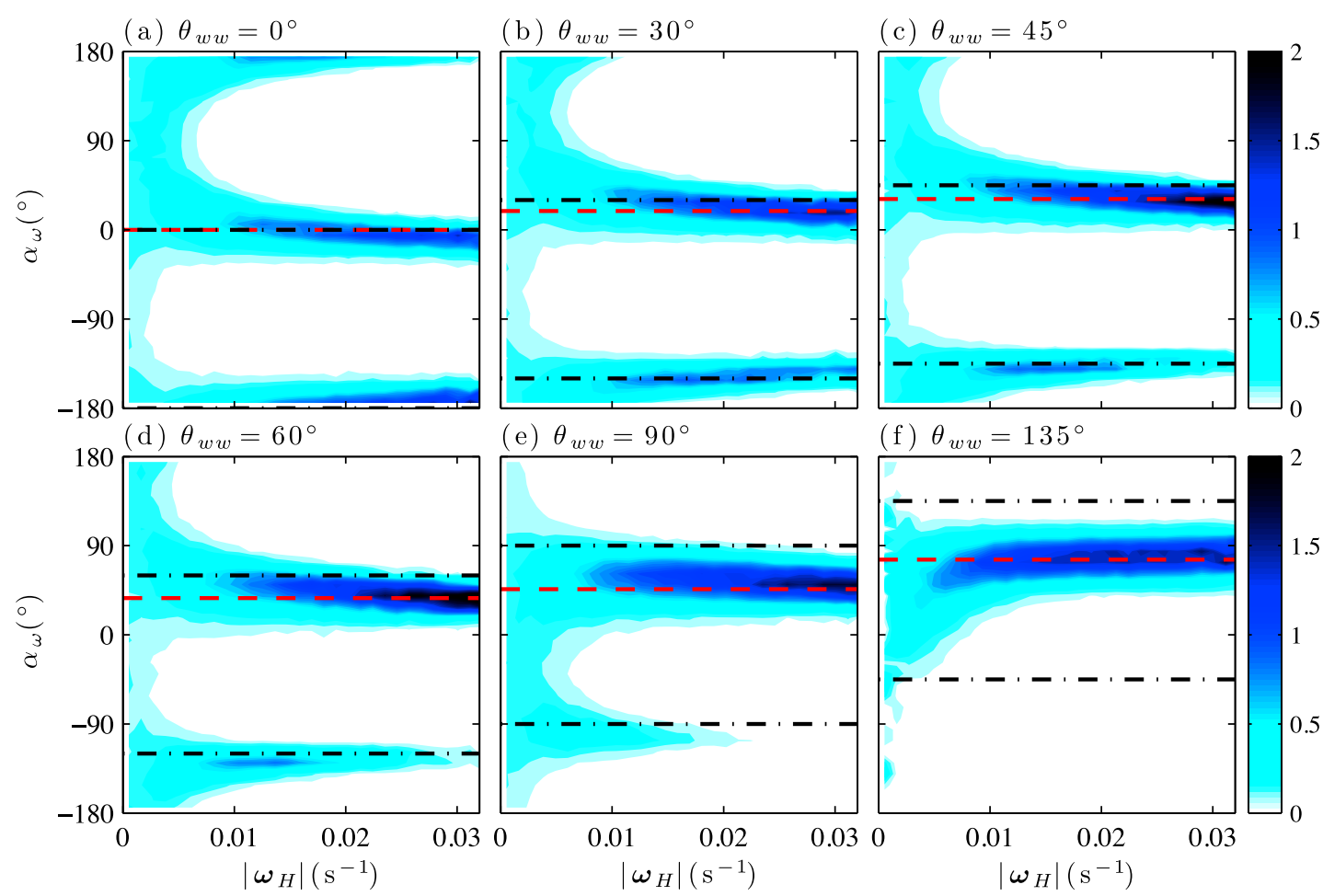

(e) $\theta_{w w}=90^{\circ}$

(f) $\theta_{w w}=135^{\circ}$
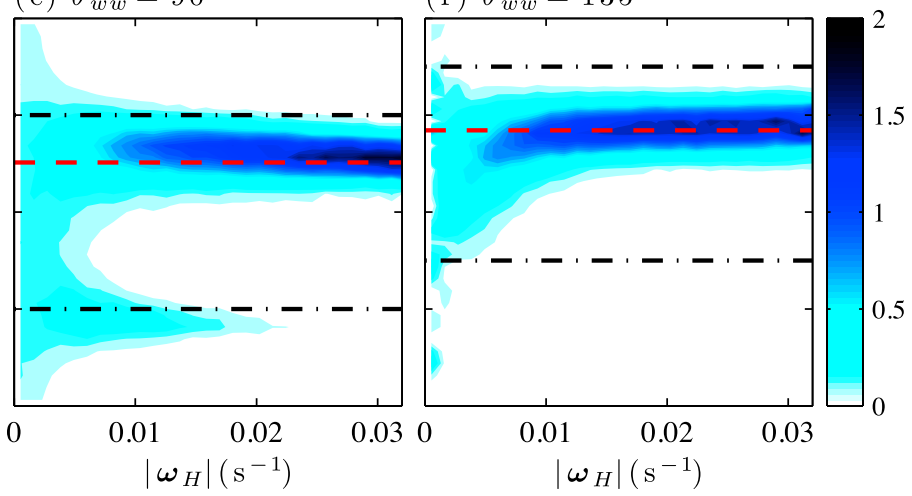

Figure 4. (a-f) Conditional pdfs $P\left(\alpha_{\omega},\left|\omega_{H}\right|\right) / P\left(\left|\omega_{H}\right|\right)$ calculated at $z=-1.5 \mathrm{~m}$ in non-rotating $\left(f=0 \mathrm{~s}^{-1}\right)$ simulations for $\theta_{w w}=0^{\circ}-135^{\circ}$. The dashed red lines are the values of $\alpha$ obtained by averaging $\alpha_{\omega}$ over the top 50\% of $\left|\boldsymbol{\omega}_{H}\right|$ values (also listed in Table 3) and the dash-dotted black lines correspond to $\theta_{w w}$ and $-180^{\circ}+\theta_{w w}$. Normalization of the pdfs is carried out for $\alpha_{\omega}=[-\pi, \pi]$.

profiles for the rotating and non-rotating cases, the nonrotating profiles do have slightly larger magnitudes than those for the rotating cases. For all cases where $\theta_{w w} \leq 90^{\circ}$, the profiles of $\overline{w^{\prime 2}}$ are substantially greater than the profile for the shear only case. The increased level of $\overline{w^{\prime 2}}$ is due to the presence of LT, and indicates that substantial turbulence production by the Stokes shear occurs even for $\theta_{w w}=$ $90^{\circ}$. As $\theta_{w w}$ continues to increase, however, there is increasingly weak production of $\overline{w^{\prime 2}}$. By $\theta_{w w}=135^{\circ}$, the observed profiles of $\overline{w^{\prime 2}}$ are only slightly different from the profiles found in the shear only case.

[24] Due to the connection between windrows and LC, there is an approximate correspondence between the angle associated with the horizontal vorticity, $\omega_{H}=\omega_{x} \hat{\mathbf{x}}+\omega_{y} \hat{\mathbf{y}}$, and the direction of the windrows. In particular, the upwelling and downwelling regions are due to the vorticity associated with LC, and the direction of LC can be diagnosed quantitatively by calculating the most likely direction of $\boldsymbol{\omega}_{H}$ in fields such as those shown in Figure 2. The angle between the LC and wind can be calculated from the statistics of the local and instantaneous direction of $\boldsymbol{\omega}_{H}$, which is given by

$$
\alpha_{\omega}=\tan ^{-1}\left(\frac{\omega_{y}}{\omega_{x}}\right)
$$

Figure 4 shows conditional probability density functions (pdfs), $P\left(\alpha_{\omega},\left|\boldsymbol{\omega}_{H}\right|\right) / P\left(\left|\boldsymbol{\omega}_{H}\right|\right)$, of $\alpha_{\omega}$ versus the horizontal vorticity magnitude, $\left|\omega_{H}\right|=\left[\omega_{x}^{2}+\omega_{y}^{2}\right]^{1 / 2}$, for the simulations without Coriolis rotation. The pdfs are calculated in $x-y$ planes near the depth of maximum VKE $(z=$ $-1.5 \mathrm{~m}$, see Figure 3). Both $\alpha_{\omega}$ and $\left|\boldsymbol{\omega}_{H}\right|$ in Figure 4 have been computed with the vertical shear of the mean horizontal currents included.

[25] Figure 4 shows that there is a primary peak in the distributions of $\alpha_{\omega}$ between $\theta_{w w}$ and $0^{\circ}$. A much weaker secondary peak is observed near $-180^{\circ}+\theta_{w w}$. A characteristic angle for the Langmuir cells can be obtained from the location of the primary peaks in Figure 4. This angle is calculated by averaging $\alpha_{\omega}$ over the largest $50 \%$ of horizontal vorticity magnitudes; we take this angle to be the true direction of Langmuir cells, and call it $\alpha$ in the following. Table 3 shows that $\alpha$ increases with $\theta_{w w}$. In particular, the LC are oriented roughly halfway between the wind and wave directions for this magnitude of $U_{s}(0)$ and $u_{*}$. The results in Table 3 also show that the inclusion of the Coriolis force slightly reduces $\alpha$, indicating a weak turning of LC orientation in the cyclonic sense in the presence of the Ekman spiral. The values of $\alpha$ from Table 3 are shown in Figure 2, and it can be seen that there is an approximate

Table 3. Mean and Standard Deviation of $\alpha_{\omega}$ From the Conditional pdfs in Figure 4 and From Rotating Versions of Similarly Forced Simulations

\begin{tabular}{lcc}
\hline$\theta_{w w}$ & Non-rotating $\left(f=0 \mathrm{~s}^{-1}\right)$ & Rotating $\left(f=10^{-4} \mathrm{~s}^{-1}\right)$ \\
\hline $30^{\circ}$ & $19^{\circ} \pm 3.2^{\circ}$ (Figure 4b) & $15^{\circ} \pm 2.7^{\circ}$ \\
$45^{\circ}$ & $31^{\circ} \pm 2.5^{\circ}$ (Figure 4c) & $25^{\circ} \pm 2.9^{\circ}$ \\
$60^{\circ}$ & $37^{\circ} \pm 2.2^{\circ}$ (Figure 4d) & $32^{\circ} \pm 2.5^{\circ}$ \\
$90^{\circ}$ & $46^{\circ} \pm 2.9^{\circ}$ (Figure 4e) & $41^{\circ} \pm 3.6^{\circ}$ \\
$135^{\circ}$ & $76^{\circ} \pm 4^{\circ}$ (Figure 4f) & $67^{\circ} \pm 3.8^{\circ}$ \\
\hline
\end{tabular}



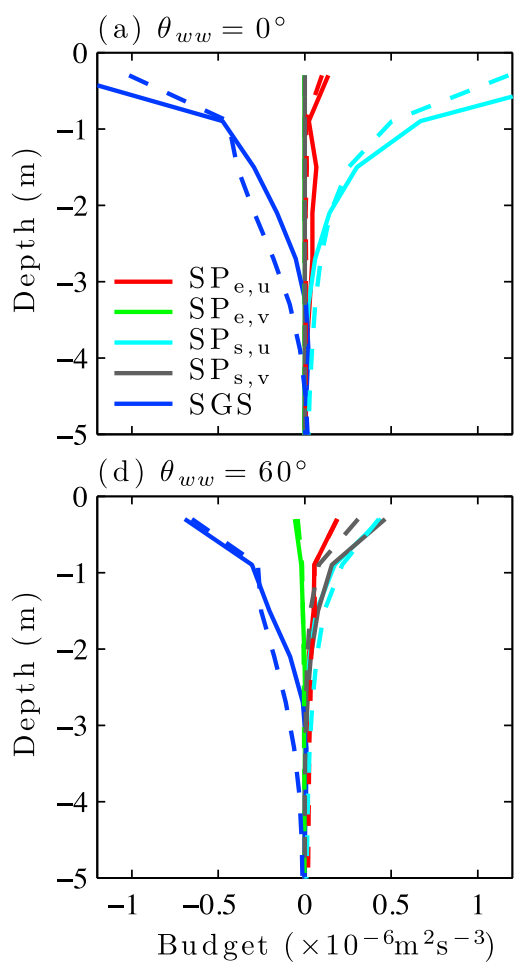

(b) $\theta_{w w}=30^{\circ}$

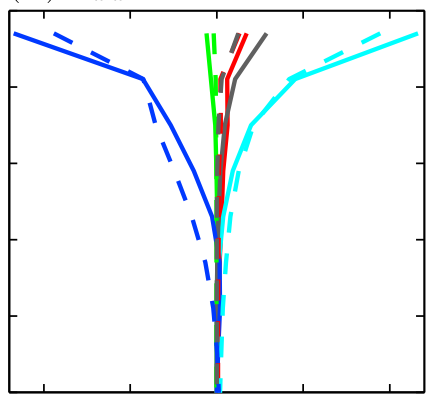

(e) $\theta_{w w}=90^{\circ}$

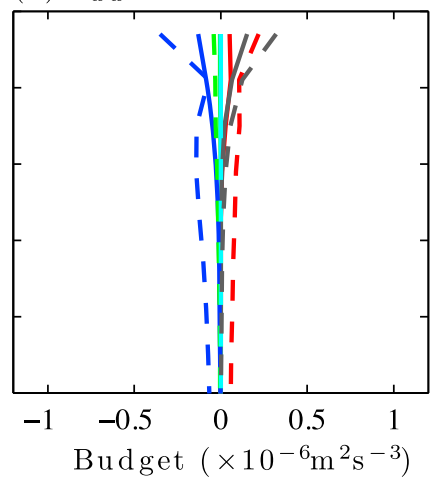

(c) $\theta_{w w}=45^{\circ}$

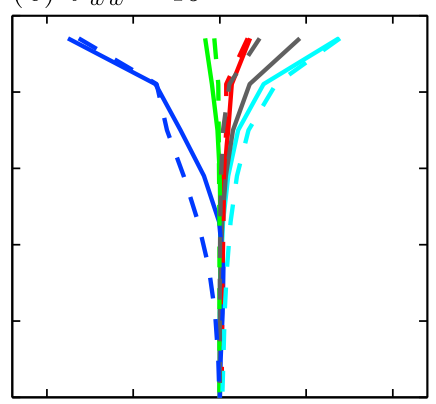

(f) $\theta_{w w}=135^{\circ}$

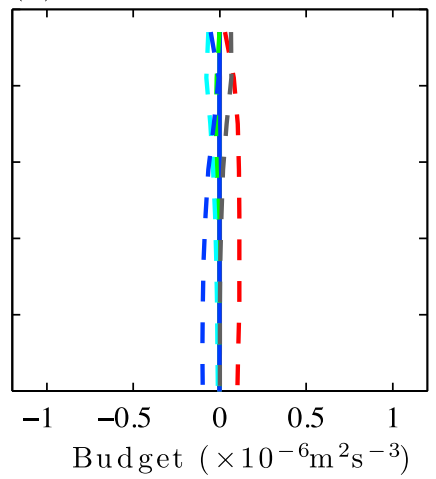

Figure 5. (a-f) Vertical profiles of TKE production and SGS terms in (7) for $\theta_{w w}=0^{\circ}-135^{\circ}$ in the nonrotating case. Solid lines are terms averaged over 20 time steps at the beginning of each simulation near the time of maximum $\mathrm{SP}_{\mathrm{s}, \mathrm{v}}$, and dashed lines are the averages once the budget terms become stationary. The legend is shown in Figure 5a, and the line labels correspond to the terms in (7).

correspondence between $\alpha$ and the direction of the windrows.

[26] Note that, for $\theta_{w w}=90^{\circ}$ and $135^{\circ}$, the primary peaks in Figures $4 \mathrm{e}$ and $4 \mathrm{f}$ become increasingly close to $\alpha_{\omega}=90^{\circ}$. This is due in part to the decreasing strength of LT for these cases (consistent with the VKE profiles in Figure 3) and the increasing influence of the mean Eulerian shear in determining the direction of $\boldsymbol{\omega}_{H}$.

[27] Figure 4 indicates that the spread of $\alpha_{\omega}$ about $\alpha$ also increases with $\theta_{w w}$, consistent with a fluctuating vorticity field. Table 3 provides error estimates of the calculated values of $\alpha$, with error bars equal to $\pm \sigma / \sqrt{N_{f}}$, where $\sigma$ is the standard deviation of $\alpha_{\omega}$ in the top $50 \%$ of horizontal vorticity magnitudes, and $N_{f}$ is the number of degrees of freedom. The approximate number of LC pairs across the domain (see Figure 2) are counted to give $N_{f} \approx 20$. For most values of $\theta_{w w}$, Table 3 shows that the error in the measured value of $\alpha$ is approximately $2^{\circ}-4^{\circ}$.

\section{Kinetic Energy and Vorticity Budgets}

\subsection{Turbulence Kinetic Energy Budget}

[28] Following several prior studies [e.g., Grant and Belcher, 2009], effects leading to the creation and maintenance of LT can be examined through an analysis of the turbulence kinetic energy (TKE) budget. Certain simplifications to this budget are possible due to the particular configuration of the present problem. Since the flow is homogeneous in the $x-y$ plane and there is no mean vertical velocity, $\partial \bar{u} / \partial z$ and $\partial \bar{v} / \partial z$ are the only nonzero components of the mean velocity gradient tensor. Here, $(u, v, w)$ denote Eulerian velocities in the $x, y$, and $z$ directions, respectively. The Stokes drift velocities, denoted $\left(u_{s}, v_{s}\right)$, also lie completely in the $x-y$ plane and again vary only in the $z$ direction. With these simplifications, and also taking advantage of the $x-y$ homogeneity of all statistics, the relevant form of the TKE equation is obtained from (1)-(4) as

$$
\begin{aligned}
\frac{\partial \bar{e}}{\partial t}+\underbrace{\frac{\partial \overline{w^{\prime} e}}{\partial z}=}_{\mathrm{TT}}= & -\underbrace{\overline{u^{\prime} w^{\prime}} \frac{\partial \bar{u}}{\partial z}}_{\mathrm{SP}_{\mathrm{e}, \mathrm{u}}}-\underbrace{\overline{v^{\prime} w^{\prime}} \frac{\partial \bar{v}}{\partial z}}_{\mathrm{SP}_{\mathrm{e}, \mathrm{v}}}-\underbrace{\overline{u^{\prime} w^{\prime}} \frac{\partial u_{s}}{\partial z}}_{\mathrm{SP}_{\mathrm{s}, \mathrm{u}}}-\underbrace{\overline{v^{\prime} w^{\prime}} \frac{\partial v_{s}}{\partial z}}_{\mathrm{SP}_{\mathrm{s}, \mathrm{v}}} \\
& +\underbrace{\frac{\partial \overline{w^{\prime} \pi^{\prime}}}{\partial z}}_{\mathrm{PT}}+\underbrace{\overline{w^{\prime} b^{\prime}}}_{\mathrm{BP}}+\mathrm{SGS},
\end{aligned}
$$

where $e=\mathbf{u}^{\prime} \cdot \mathbf{u}^{\prime} / 2$ is the TKE and $b^{\prime}=-g \rho^{\prime} / \rho_{0}$ is the fluctuating buoyancy. Fluctuating variables are defined as $\phi^{\prime} \equiv$ $\phi-\bar{\phi}$. In (7), TT denotes the turbulent transport of TKE, $\mathrm{SP}_{\mathrm{e}, \mathrm{u}}$ and $\mathrm{SP}_{\mathrm{e}, \mathrm{v}}$ are the Eulerian shear production by the $u$ and $v$ shear components, respectively, $\mathrm{SP}_{\mathrm{s}, \mathrm{u}}$ and $\mathrm{SP}_{\mathrm{s}, \mathrm{v}}$ are the $u$ and $v$ components, respectively, of the shear production due to the Stokes drift, PT is the pressure transport, and SGS denotes subgrid-scale terms, including kinetic energy dissipation. Buoyant production, BP, of TKE is minimal in the simulations and is thus ignored.

[29] Figure 5 shows the shear production and SGS terms in the TKE budgets for $\theta_{w w}=0^{\circ}-135^{\circ}$ in the non-rotating case (the budgets for the rotating cases are similar). The statistics have been averaged over two time intervals. The 

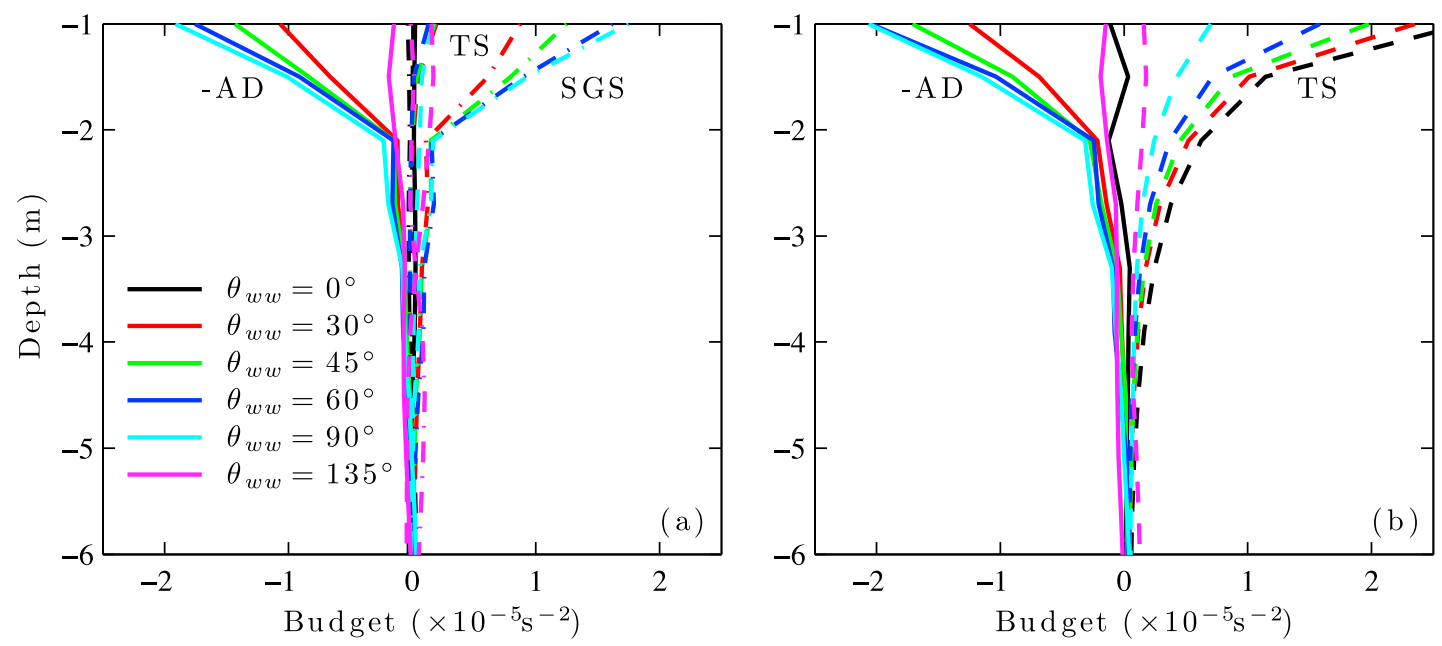

Figure 6. Budgets of along-cell vorticity, $\xi$, for $\theta_{w w}=0^{\circ}-135^{\circ}$ in the non-rotating case. Lines correspond to terms in (9); - AD (solid lines), TS (dashed lines) and SGS (dash-dotted lines). (a) Budget terms averaged over the full domain and (b) budget terms averaged only over points where $\xi>0$ are shown. SGS terms are not shown in Figure 6b.

first interval is at the beginning of each run near the time of maximum production of TKE by the Stokes drift production in the $y$-direction, namely $\mathrm{SP}_{\mathrm{s}, \mathrm{v}}$. The second averaging interval spans a much longer time after the turbulence statistics have become stationary. Analysis of these two intervals is important, since it appears that the direction of the Langmuir cells is set during the growth phase and that they then persist in that direction unabated by the change in the direction dominating the shear production. The SGS terms have been calculated from the averages of the various terms in (7) by assuming that $\partial \bar{e} / \partial t$ is approximately zero.

[30] Figure 5 shows that the TKE budgets undergo significant changes as $\theta_{w w}$ increases. For small $\theta_{w w}$, the primary balance is between the Stokes shear production in the $x$-direction, $\mathrm{SP}_{\mathrm{s}, \mathrm{u}}$, and the $\mathrm{SGS}$ terms. As $\theta_{w w}$ increases, however, $\mathrm{SP}_{\mathrm{s}, \mathrm{u}}$ decreases, until it is essentially 0 at $\theta_{w w}=90^{\circ}$ (Figure 5e). At the same time, the Stokes shear production in the $y$-direction, $\mathrm{SP}_{\mathrm{s}, \mathrm{v}}$, increases weakly as $\theta_{w w}$ increases. The remaining, non-Stokes, terms in the budgets remain relatively constant for all $\theta_{w w}$, except for $\theta_{w w}=135^{\circ}$ where all terms become small.

[31] The turbulent momentum flux $\overline{\mathbf{u}^{\prime} w^{\prime}}$ near the end of the simulation points in a direction biased toward the wind. That is, the turbulent momentum flux produced by the LES model after the onset and stabilization of LT is dominated by shear instability induced by the wind stress, even though the LC dominate the VKE. A TKE budget taken from near the time of maximum $\mathrm{SP}_{\mathrm{s}, \mathrm{v}}$ compared to near the end of the run shows that $\mathrm{SP}_{\mathrm{s}, \mathrm{v}}$ generally decreases after LT onset.

[32] The time-dependence of the TKE budget analysis leaves uncertainty as to the mechanisms by which the LC are maintained after their onset. The energy production that results in the direction of the Langmuir cells is dominant only during the initial growth phase of LT. After this period, the turbulence continues to grow and TKE production becomes increasingly dominated by the shear in the wind direction, even though the direction of the Langmuir cells remains unchanged.

\subsection{Vorticity Budget}

[33] Although the TKE budget gives insights into the energetics associated with the creation of LT, it does not directly allow an analysis of the dynamical processes maintaining the orientation and strength of LC. To understand these processes, we consider the budget equation for the absolute vorticity, $\boldsymbol{\omega}_{a}=\boldsymbol{\omega}+f \hat{\mathbf{z}}$, which can be found from the curl of the CL momentum equation in (3) as

$$
\frac{\partial \boldsymbol{\omega}_{a}}{\partial t}+\underbrace{\left(\mathbf{u}_{L} \cdot \nabla\right) \boldsymbol{\omega}_{a}}_{\mathrm{AD}}=\underbrace{\left(\boldsymbol{\omega}_{a} \cdot \nabla\right) \mathbf{u}_{L}}_{\mathrm{TS}}+\underbrace{\nabla b \times \hat{\mathbf{z}}}_{\mathrm{BV}}+\mathrm{SGS},
$$

where $b=-g \rho / \rho_{0}$ is the buoyancy. Here, AD is vortex advection, TS is vortex tilting/stretching, and SGS again denotes subgrid-scale terms. The buoyancy term (BV) was determined to be small and is ignored in the following budgets. Note that, contrary to the transport equation for $\boldsymbol{\omega}$ from the Navier-Stokes equations, the Lagrangian velocity $\mathbf{u}_{L}=\mathbf{u}+\mathbf{u}_{s}$ appears in the advection and vortex stretching terms in (8).

[34] In the following, we will focus on the along-LC vorticity, $\xi=\omega_{a} \cdot \hat{x}^{\prime}$, where $\hat{x}^{\prime}$ is the unit vector in the alongLC direction, and $f \hat{\mathbf{z}} \cdot \hat{x}^{\prime}=0$ (see Figure 1). The cross-cell vorticity components are denoted by $\eta$ and $\zeta$, corresponding to the $\hat{y}^{\prime}$ and $\hat{\mathbf{z}}$ directions, respectively. The vorticity equation for $\xi$ is given from (8) as [e.g., Holm, 1996]

$$
\frac{\partial \xi}{\partial t}+\underbrace{\left(\mathbf{u}_{L} \cdot \nabla\right) \xi}_{\mathrm{AD}}=\underbrace{\left(\boldsymbol{\omega}_{a} \cdot \nabla\right)\left(\mathbf{u}_{L} \cdot \hat{\mathbf{x}}^{\prime}\right)}_{\mathrm{TS}}+\underbrace{(\nabla b \times \hat{\mathbf{z}}) \cdot \hat{\mathbf{x}}^{\prime}}_{\mathrm{BV}}+\mathrm{SGS},
$$

where the labels for each term are the same as in (8).

[35] Using the angle of the Lagrangian shear, denoted $\alpha_{\mathcal{L}}$ (which will be discussed in more detail in section 5) to determine the direction of $\hat{x}^{\prime}$, profiles of the terms in (9), averaged over the full domain, are shown in Figure 6a. The 

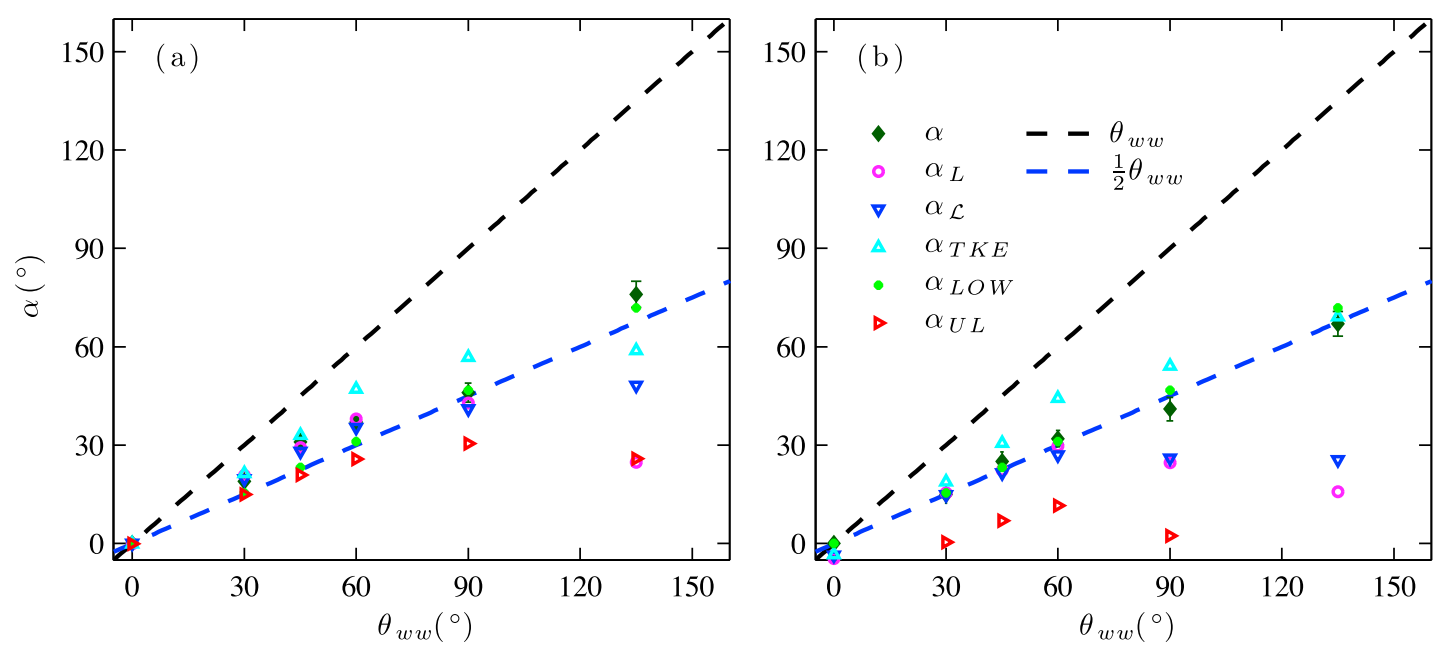

Figure 7. Observed and predicted values of $\alpha$ as a function of $\theta_{w w}$ for (a) non-rotating and (b) rotating simulations. The angles shown are: the vorticity-diagnosed $\alpha$ from Table 3, the Lagrangian shear angle $\alpha_{L}$ from (10), the depth-averaged TKE/Lagrangian shear angle $\alpha_{\mathcal{L}}$ from (14), the depth-averaged TKE angle $\alpha_{T K E}$ from (12), the law of the wall prediction $\alpha_{L O W}$ from (18), and the mean Lagrangian velocity direction near the surface, $\alpha_{U L}$. The error bars on $\alpha$ are obtained from Table 3 . Included in both figures are lines showing $\alpha=\theta_{w w}$ (black dashed line) and $\alpha=\theta_{w w} / 2$ (blue dashed line).

terms in these budgets supply the creation of LC and prove to be revealing as to how the misaligned cases differ from traditional, aligned LC. The SGS terms are calculated from the domain averages of $\mathrm{AD}$ and TS by assuming that the domain average of $\partial \xi / \partial t$ is zero. In the aligned case $\left(\theta_{w w}=\right.$ $0^{\circ}$ ), full domain averages of the AD, TS, and SGS terms are all approximately zero. In this case, the positive and negative vortices associated with each LC pair are approximately equivalent in magnitude and size. Therefore, when the terms are averaged over the domain (with periodic boundary conditions), the result is nearly zero.

[36] When the waves are misaligned with the wind stress, asymmetry is introduced and the averaged budget terms no longer vanish. Figure 6a shows that, for $\theta_{w w}>0^{\circ}, \mathrm{AD}$ balances the SGS terms, with TS playing a secondary role. As $\theta_{w w}$ increases, the magnitudes of the AD and SGS terms increase. This indicates, in particular, that there is significant cross-cell vorticity advection. This advection has two apparent effects. First, the cross-cell Eulerian advection transports axial vorticity from the upwind cell onto the downwind, which introduces an asymmetry. Second, the cross cell velocity seems to enhance a secondary alongstream instability that leads to coherent cells that are shorter in the along-LC direction ( $x^{\prime}$; Figure 1). These instabilities are evident in horizontal slices of vertical velocity (see Figure 2). As $\theta_{w w}$ increases, the variability of vertical velocity along the LC direction increases, and the anisotropy of the velocity and vorticity statistics decreases (Figure 4). The dependence of LC direction and strength on the cross cell advection is in general agreement with the hypothesis by Gnanadesikan and Weller [1995] that the LC will minimize cross-cell shear while maximizing the wave-current interaction. The $\mathrm{AD}$ and SGS terms continue to increase in magnitude up to $\theta_{w w}=90^{\circ}$, before decreasing again at $\theta_{w w}=135^{\circ}$.

[37] Note that, since LC tend to occur in pairs of oppositely-signed vortices, there can be difficulties in using full domain averages for vorticity budget analysis. Consequently, Figure $6 \mathrm{~b}$ shows the $\mathrm{AD}$ and TS terms from (9) averaged only over points where $\xi>0$. Again the AD terms become larger in magnitude as $\theta_{w w}$ increases (with a subsequent decrease for $\theta_{w w}=135^{\circ}$ ), but now the TS terms are also nonzero. These results will be examined in more detail in section 6 , where the conditional averaging procedure of Kukulka et al. [2010] is used to analyze the vorticity budget of a composite LC vortex pair.

\section{Analysis of Langmuir Cell Orientation}

[38] As a prelude to formulating a priori predictions of $\alpha$ based on the Stokes drift profile and other problem parameters, we can consider the values of $\alpha$ predicted through analyses of the TKE and vorticity budgets in the previous section. The orientations of LC are fundamentally governed by the dynamics of the vorticity transport equations in (8) and (9). In particular, the mechanism for production of along-LC vorticity is the tilting of vertical vorticity by the Lagrangian shear [Leibovich, 1983]. The angle of the mean Lagrangian shear, denoted $\alpha_{L}$, thus provides a natural direction for $\mathrm{LC}$ and is given by

$$
\tan \left(\alpha_{L}\right)=\frac{\left(\partial \bar{v}_{L} / \partial z\right)}{\left(\partial \bar{u}_{L} / \partial z\right)}=\frac{(\partial \bar{v} / \partial z)+\left(\partial v_{s} / \partial z\right)}{(\partial \bar{u} / \partial z)+\left(\partial u_{s} / \partial z\right)} .
$$

This angle is shown as a function of $\theta_{w w}$ in Figure 7. Since $\alpha_{L}$ is generally a function of $z$, we show $\alpha_{L}$ at $z=-1.5 \mathrm{~m}$, corresponding once again to the depth of maximum $\overline{w^{\prime 2}}$. Figure 7 shows that $\alpha_{L}$ is in relatively good agreement with $\alpha$ (from Table 3) for $\theta_{w w}<90^{\circ}$. At $\theta_{w w}=90^{\circ}$ and $135^{\circ}$, however, $\alpha_{L}$ is substantially less than $\alpha$. This difference is due, in part, to the bias introduced by the mean $\boldsymbol{\omega}$ as the strength of LT decreases with increasing $\theta_{w w}$. For sufficiently weak LC, such as occurs for large $\theta_{w w}$, the mean $\boldsymbol{\omega}$ 
makes an angle of $90^{\circ}$ with the $x$-axis, thus biasing $\alpha$ toward larger values.

[39] The TKE budget can also be used to obtain an LC angle similar to $\alpha_{L}$. Defining the direction of $\overline{\mathbf{u}^{\prime} w^{\prime}}$ relative to the wind by the new angle $\alpha_{l}$, an expression for $\alpha_{l}$ can be obtained by assuming that the flow evolves such that the total TKE production, $\mathrm{SP}_{\mathrm{e}}+\mathrm{SP}_{\mathrm{s}}$, is maximized. This total production is simply the Lagrangian shear production, and can be written in terms of $\alpha_{l}$ and other parameters as

$$
\overline{\mathbf{u}^{\prime} w^{\prime}} \cdot \frac{\partial \overline{\mathbf{u}}_{L}}{\partial z}=\left|\overline{\mathbf{u}^{\prime} w^{\prime}}\right|\left|\frac{\partial \overline{\mathbf{u}}}{\partial z}\right| \cos \left(\theta_{e}-\alpha_{l}\right)+\left|\overline{\mathbf{u}^{\prime} w^{\prime}}\right|\left|\frac{\partial \mathbf{u}_{s}}{\partial z}\right| \cos \left(\theta_{w w}-\alpha_{l}\right),
$$

where $\theta_{e}$ is the angle between the Eulerian shear, $\partial \overline{\mathbf{u}} / \partial z$, and the wind. Finding the value of $\alpha_{l}$ which maximizes the production yields

$$
\tan \left(\alpha_{l}\right)=\frac{|\partial \overline{\mathbf{u}} / \partial z| \sin \theta_{e}+\left|\partial \mathbf{u}_{s} / \partial z\right| \sin \theta_{w w}}{|\partial \overline{\mathbf{u}} / \partial z| \cos \theta_{e}+\left|\partial \mathbf{u}_{s} / \partial z\right| \cos \theta_{w w}} .
$$

The numerator is simply the cross-wind $(y)$ component of the Lagrangian shear, while the denominator is the corresponding along-wind $(x)$ component, and the angles $\alpha_{L}$ and $\alpha_{l}$ are related by

$$
\tan \left(\alpha_{l}\right)=\frac{\left(\partial \bar{v}_{L} / \partial z\right)}{\left(\partial \bar{u}_{L} / \partial z\right)}=\tan \left(\alpha_{L}\right)
$$

Thus, the directions obtained from vortex stretching and maximization of TKE production are identical.

[40] The Lagrangian shear, $\partial \overline{\mathbf{u}}_{L} / \partial z$, in (10) is a function of depth, $z$, and thus the angle $\alpha_{L}$ is also a function of $z$. In order to obtain a single value for the direction of $\mathrm{LC}$, we can consider an angle averaged over some depth, $D_{l}$. We can thus define a new, depth-averaged angle, $\alpha_{\mathcal{L}}$, as

$$
\tan \left(\alpha_{\mathcal{L}}\right)=\frac{\left\langle\partial \bar{v}_{L} / \partial z\right\rangle_{D_{l}}}{\left\langle\partial \bar{u}_{L} / \partial z\right\rangle_{D_{l}}}
$$

From (12) we can also write

$$
\tan \left(\alpha_{T K E}\right)=\frac{\left\langle\left|\overline{\mathbf{u}^{\prime} w^{\prime}}\right|\left(\partial \bar{v}_{L} / \partial z\right)\right\rangle_{D_{l}},}{\left\langle\overline{\mathbf{u}^{\prime} w^{\prime}} \mid\left(\partial \bar{u}_{L} / \partial z\right)\right\rangle_{D_{l}}}
$$

where $\left|\overline{\mathbf{u}^{\prime} w^{\prime}}\right|$ is included in (15) in order to examine the depth-average ratio of the full $x$ and $y$ production terms. The volume average $\langle\cdot\rangle_{D_{l}}$ to depth $D_{l}$ in (14) and (15) is defined as

$$
\langle f\rangle_{D_{l}} \equiv \frac{1}{L_{x} L_{y}\left|D_{l}\right|} \int_{0}^{L_{x}} \int_{0}^{L_{y}} \int_{D_{l}}^{0} f(x, y, z) \mathrm{d} x \mathrm{~d} y \mathrm{~d} z .
$$

Here, $D_{l}$ is usually taken as twice the $e$-folding depth of the Stokes drift due to the peak frequency of the wave spectrum. If $D_{l} \approx-6 \mathrm{~m} \approx 0.2 H_{M L}$, this depth is nearly equivalent to the depth below which all of the TKE production terms are negligible (see Figure 5). Figure 7 shows that, using the full shear profile from LES, $\alpha_{\mathcal{L}}$ and $\alpha_{T K E}$ are both similar to $\alpha$ for angles smaller than $90^{\circ}$. In the following, $\alpha_{\mathcal{L}}$ is used as the LC angle.

\subsection{Predictions of Cell Direction}

[41] Determining the angles discussed in the previous section requires statistics from LES. For parameterizations and climatologies of LT, these statistics are not available, and so predictions for $\alpha$ that do not require LES results are useful. Since the orientation of LC depends on the direction of Lagrangian shear, it is natural to take the depth-averaged Lagrangian shear angle in (14) as a starting point. Assuming that the Stokes shear components of the Lagrangian shear are known quantities from wave data or models [Webb and Fox-Kemper, 2011], the only unknown in (14) is the direction of the Eulerian shear. In the previous section, the Eulerian shear is measured from the LES, but we can obtain an a priori prediction for the cell direction by assuming that the Eulerian shear can be approximated by the law of the wall, namely

$$
\frac{\partial \bar{u}}{\partial z} \approx-\frac{u_{*}}{\kappa z} \Rightarrow \frac{\partial \overline{\mathbf{u}}_{L}}{\partial z} \approx-\frac{u_{*}}{\kappa z} \hat{\mathbf{x}}+\frac{\partial \mathbf{u}_{s}}{\partial z},
$$

where $\kappa$ is the von Kármán constant and $z$ is a depth below the surface. This expression assumes that the cross-wind (y-direction) Eulerian shear is negligible, and that the Stokes shear can be computed from wave data. The resulting law of the wall based angle, denoted $\alpha_{L O W}$, is then given using (14) as

$$
\tan \left(\alpha_{L O W}\right)=\frac{\left\langle\partial v_{s} / \partial z\right\rangle_{D_{l}}}{\frac{u_{*}}{\kappa\left|z_{1}-D_{l}\right|} \ln \left(\left|D_{l} / z_{1}\right|\right)+\left\langle\partial u_{s} / \partial z\right\rangle_{D_{l}}} .
$$

In this equation, the first term in the denominator is obtained by integrating the law of the wall approximation for $\partial \bar{u} / \partial z$ over the range $z=\left[D_{l}, z_{1}\right]$ using (16), where $z_{1}$ is a depth slightly below the surface (e.g., the first grid point of the LES, or the significant wave height).

[42] Figure 7 shows $\alpha_{\text {LOW }}$ from (18) calculated using $D_{l}=-6 \mathrm{~m}$. Once again, this is the depth below which the TKE production terms in Figure 5 are negligible. Figure 7 shows that $\alpha_{L O W}$ falls within or near the error bars of $\alpha$ for all $\theta_{w w}$. Interestingly, $\alpha_{L O W}$ is equally accurate for both the non-rotating and rotating cases, despite the presence of Ekman spiral shear in the rotating cases.

[43] Overall, $\alpha, \alpha_{\mathcal{L}}, \alpha_{T K E}$, and $\alpha_{L O W}$ agree for $\theta_{w w}<90^{\circ}$. For the most extreme case $\left(\theta_{w w}=135^{\circ}\right)$, however, $\alpha_{\mathcal{L}}$ underestimates $\alpha$. These underestimates are most likely accurate, since Figure 3 shows that $\overline{w^{\prime 2}}$ is close to the shearonly result for $\theta_{w w}=135^{\circ}$, and Figure 8 shows that rescaling with $\alpha_{L O W}$ tends to overestimate the VKE in the $\theta_{w w}=135^{\circ}$ case. These results show that Langmuir mixing is very weak when $\theta_{w w}>90$. If there is no Langmuir mixing, then waves are ineffective at generating LC, and the desired angle should go to zero (turbulence alignment with wind only). However, the result using $\alpha_{L O W}$ in Figure 8 is not infinite or poorly posed, and these nearly-antiparallel situations would be transient in reality. Thus, we do not expect the application of $\alpha_{L O W}$, which is accurate for all cases where $\theta_{w w} \leq 90^{\circ}$, to be overly biased. If this is a critical concern in understanding a particular data set, LES may be used to determine $\alpha_{\mathcal{L}}$ for anomalous situations rather than relying on $\alpha_{L O W}$. This case will be discussed in more detail in section 6 . 

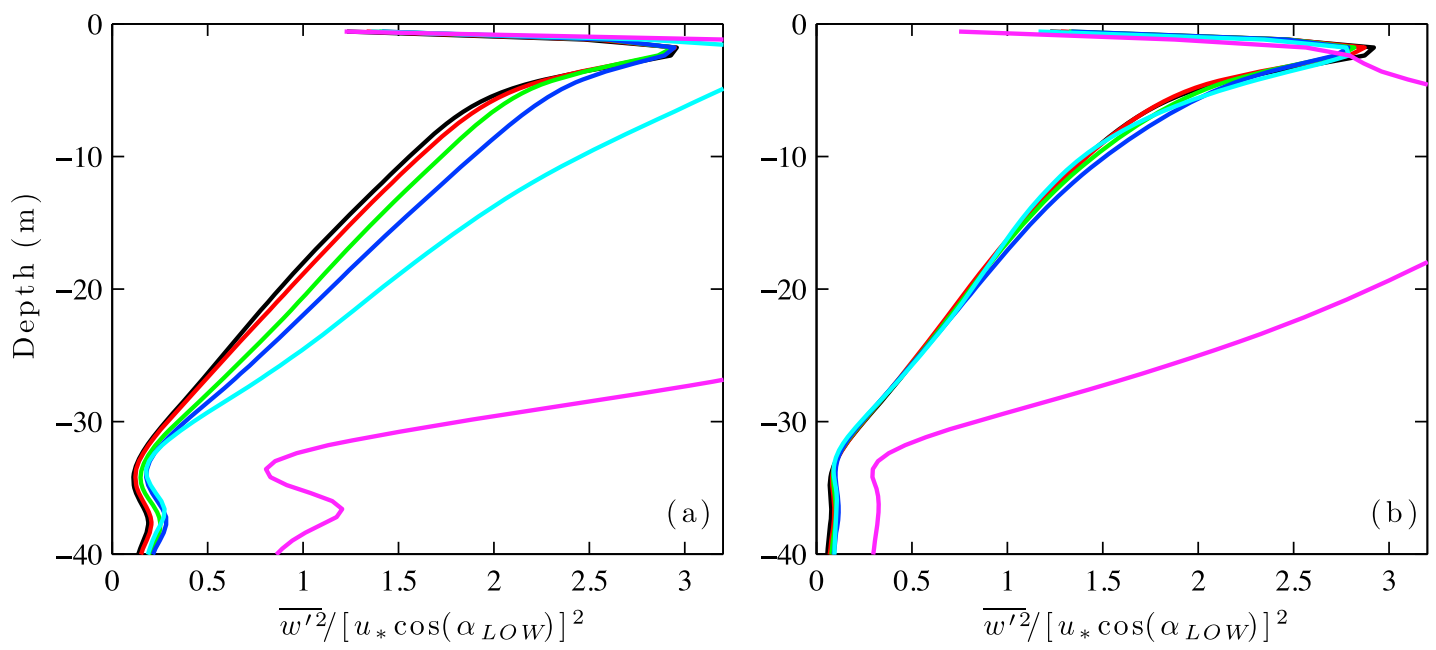

Figure 8. Vertical profiles of $\overline{w^{\prime 2}}$ normalized by $\left[u_{*} \cos \left(\alpha_{L O W}\right)\right]^{2}$, where $\alpha_{L O W}$ is given in (18), for (a) non-rotating and (b) rotating cases. Results are shown for $\theta_{w w}=0^{\circ}-135^{\circ}$, and legend is shown in Figure 3a.

\subsection{Other Predictions of Cell Direction}

[44] While the predictions for the direction of LC in the previous sections are based on analyses of the vorticity and TKE budgets, a number of other predictions are also possible. Perhaps the simplest prediction [Cox, 1997] assumes that LC point in the direction of either the surface stress or Stokes drift, depending on which is larger. A prediction of the corresponding angle, denoted $\alpha_{0}$, can then be written as

$$
\tan \left(\alpha_{0}\right)= \begin{cases}\frac{\tau_{y}}{\tau_{x}}, & \text { if }\left|\mathbf{u}_{*}\right| \geq\left|\mathbf{u}_{s}(0)\right| \\ \frac{v_{s}(0)}{u_{s}(0)}, & \text { if }\left|\mathbf{u}_{s}(0)\right|>\left|\mathbf{u}_{*}\right|\end{cases}
$$

where $\tau_{x}$ and $\tau_{y}$ are the wind stress components in the $x$ - and $y$-directions, respectively. In the present simulations where the wind is always parallel to the $x$-direction, $\tau_{y}=0$ and $\left|\mathbf{u}_{s}(0)\right|$ is always greater than $\left|\mathbf{u}_{*}\right|$. Moreover, the angle given by $\tan ^{-1}\left[v_{s}(0) / u_{s}(0)\right]$ is simply $\theta_{w w}$. Consequently, $\alpha_{0}=\theta_{w w}$, which is much larger than $\alpha$ for all $\theta_{w w}$ (Figure 7).

[45] An attempt at improving (19) can be made by assuming that Langmuir mixing occurs whenever a portion of the Stokes drift and surface stress project onto a third vector (the presumed LC direction) that lies somewhere between these two vectors. The angle that optimizes the projection of $\mathbf{u}_{*}$ and $\mathbf{u}_{s}(0)$ is $\alpha_{B}$, which can be shown to be

$$
\tan \left(\alpha_{B}\right)=\frac{\left|\mathbf{u}_{s}(0)\right| \sin \left(\theta_{w w}\right)}{u_{*}+\left|\mathbf{u}_{s}(0)\right| \cos \left(\theta_{w w}\right)} .
$$

This equation gives predictions of the LC orientation for any value of $\theta_{w w}<180^{\circ}$. However, since $u_{*} /\left|\mathbf{u}_{s}(0)\right|$ is small normally (roughly equal to 0.08 , see Table 1 ), $\alpha_{B} \approx \theta_{w w}$ for all $\theta_{w w}$. As a result, $\alpha_{B}$ once again substantially over-predicts the values of $\alpha$ shown in Figure 7.

[46] It is perhaps better to think of (20) as a (poor) approximation to the surface Lagrangian velocity direction. If the Eulerian mean velocity along the $x$-direction just below the surface, which is obtained from the LES, is used in place of $u_{*}$, and the Eulerian mean velocity in the $y$-direction is included in the numerator, then the resulting angle is that of the Lagrangian velocity $\overline{\mathbf{u}}_{L}$, and is denoted $\alpha_{U L}$. Figure 7 shows that, for the non-rotating cases, $\alpha_{U L}$ lies within the error bars of $\alpha$ for $\theta_{w w} \leq 30^{\circ}$, but under-predicts $\alpha$ for larger $\theta_{w w}$. For the rotating cases, the Ekman spiral causes $\alpha_{U L}$ to severely under-predict $\alpha$ for all $\theta_{w w}$. Note that the crucial difference between (20) and (18) is that the latter focuses on the Lagrangian shear direction, which was shown above to dominate both the direction of vortex tilting and TKE production. The surface Lagrangian velocity is a poor approximation to the shear direction in an Ekman spiral.

\section{Conditional Analysis of Langmuir Cells}

[47] To gain additional insights into the vorticity balance for LC, we use conditional averaging [see, e.g., McWilliams et al., 1997; Kukulka et al., 2010] to develop a picture of the flow structure near a typical LC pair. At the center of each pair is a large vertical velocity (directed either up or down), and here we identify pairs by searching for regions of strong downwelling flow. We confine our search to the depth at which $\overline{w^{\prime 2}} 1 / 2$ is maximum, denoted $z_{\max }$ (see Figure 3), and seek locations where $\left.w^{\prime}<-2\left(\overline{w^{\prime 2}}\right)^{1 / 2}\right)_{\max }$. Searching along rows of constant $x$, any cross-wind location at which this condition is satisfied, denoted $y^{k}$, is considered the center of an LC pair. The superscript $k$ is used to index each pair, and there are $n$ total pairs. We can then construct a conditional average, denoted $\bar{F}_{c o n}(y, z, t)$, as

$$
\bar{F}_{c o n}(y, z, t)=\frac{1}{n} \sum_{k=1}^{n} F\left(x^{k}, y-y^{k}, z, t\right),
$$

where $F$ is an arbitrary flow quantity. The two-dimensional planes of $\bar{F}_{c o n}(y, z, t)$ obtained from (21) are averaged crosssections of the quantity $F$ projected in the $y-z$ plane for a typical LC pair. These planes can be projected into the $y^{\prime}-z$ plane instead by noting that the $y^{\prime}-z$ plane is perpendicular to the cell axis, $x^{\prime}$, and differs from the $y-z$ plane by an 


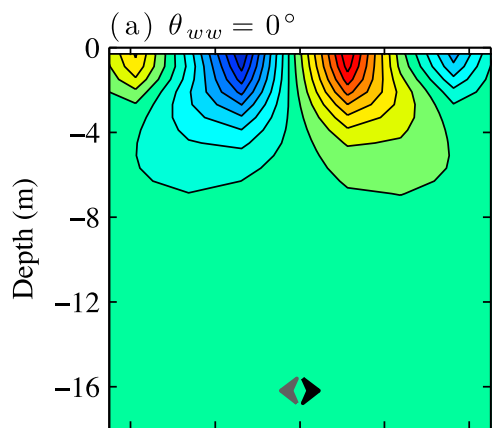

(d) $\theta_{w w}=60^{\circ}$

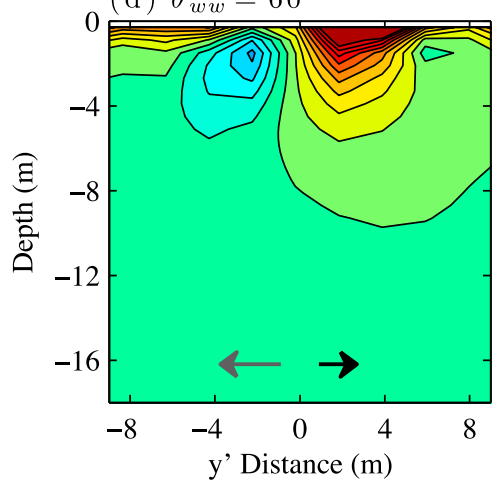

(b) $\theta_{w w}=30^{\circ}$

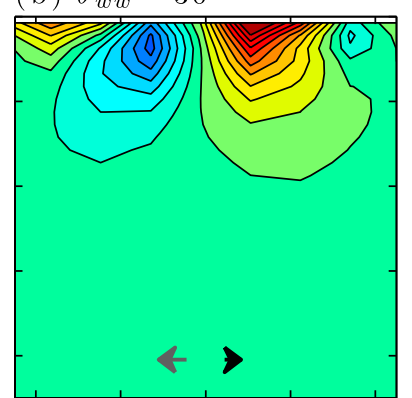

(e) $\theta_{w w}=90^{\circ}$

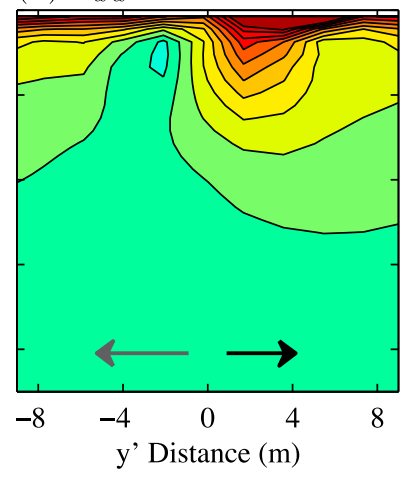

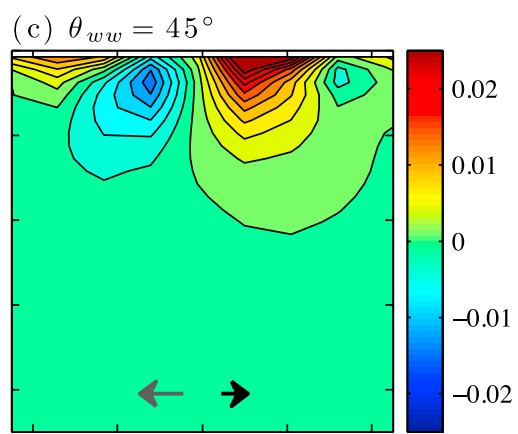

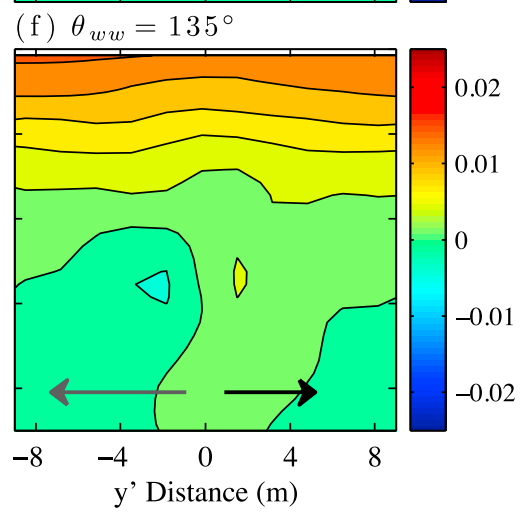

Figure 9. (a-f) Conditionally averaged axial vorticity, $\xi$, in non-rotating cases for $\theta_{w w}=0^{\circ}-135^{\circ}$. The plots are in the $\left(y^{\prime}, z\right)$ plane, with the $x^{\prime}$-axis directed out of the page, and $\xi>0$ indicates counterclockwise circulations (see Figure 1). At the bottom of the figures, the black arrows show $\mathbf{u}_{s}(0) \cdot \hat{\mathbf{y}}^{\prime}$ and the gray arrows show $\overline{\mathbf{u}}(0) \cdot \hat{\mathbf{y}}^{\prime}$. The mean cross cell velocities and Stokes drift are scaled to the $\theta_{w w}=90^{\circ}$ value of $\overline{\mathbf{u}}(0) \cdot \hat{\mathbf{y}}^{\prime}$.

angle determined by the Langmuir cell direction $\left(\alpha \approx \alpha_{\mathcal{L}}\right.$, see Figure 1).

[48] Figure 9 shows conditionally-averaged fields of the axial vorticity, $\bar{\xi}_{c o n}\left(y^{\prime}, z\right)$, for six values of $\theta_{w w}$ in the nonrotating simulations. In order to obtain the two-dimensional profiles in Figure 9, we have also averaged over time. For all $\theta_{w w}<90^{\circ}$, Figure 9 shows that the typical LC pair consists of two counter-rotating vortices. There is strong symmetry in the structure of the LC pair in the aligned simulation $\left(\theta_{w w}=0^{\circ}\right.$ in Figure 9a).This is consistent with the negligible domain-averaged terms in the $\xi$ budget shown in Figure 6; these terms average to zero due to the cancelation caused by the symmetry of the counter-rotating pair in Figure 9a. As $\theta_{w w}$ increases, Figure 9 shows that the upwind cell of each pair increases in magnitude, while the downwind cell decreases in magnitude. Here, upwind $\left(y^{\prime}>0\right)$ and downwind $\left(y^{\prime}<0\right)$ refer to the location of the cells relative to the direction of the mean Eulerian velocity (shown at the bottom of each profile). Figures $9 \mathrm{e}$ and $9 \mathrm{f}$ show that, after $\theta_{w w}$ exceeds $90^{\circ}$, vortices are no longer located by the conditional averaging procedure. This confirms the statistics from preceding sections where LC are weak or no longer present at $\theta_{w w}=135^{\circ}$.

[49] The decrease in magnitude of the downwind cell with increasing $\theta_{w w}$ in Figure 9 is consistent with cross-cell advection of positive $\xi$ from the upwind cell. The arrows on Figure 9, which indicate the strengths of cross-cell advection by Eulerian and Stokes drift velocities, provide a partial explanation of this result; as $\theta_{w w}$ increases, the cross-cell
Eulerian velocity increasingly overwhelms the Stokes drift in advecting $\xi$ from the upwind cell onto the downwind cell. However, if this were the sole mechanism acting to create the cell asymmetry shown in Figure 9, the magnitude of $\xi$ in the upwind cell would decrease. Since this decrease is not seen, $\xi$ in the upwind cell actually increases with increasing $\theta_{w w}$, we expect other terms in the vorticity budget to contribute to the increase in $\xi$.

[50] The effect of these terms can be examined through conditional averages of the $\xi$ budget terms in (9), as shown for $\theta_{w w}=0^{\circ}, 45^{\circ}$ and $90^{\circ}$ in Figure 10 . These conditional averages are again calculated using (21); the budget terms are computed at every grid point and then conditionally averaged to construct characteristic $y^{\prime}-z$ fields near LC pairs. In addition to temporal averaging, the vertical profiles in Figure 10 are obtained using an additional level of averaging. In particular, in order to determine the budget terms near the upwind and downwind cells, we also average the terms over $y^{\prime}$ for $y^{\prime}>0$ (upwind) and $y^{\prime}<0$ (downwind). This gives the two sets of one-dimensional vertical profiles in Figure 10.

[51] Figures 10a and 10b show that for the aligned case $\left(\theta_{w w}=0^{\circ}\right)$, there is a balance near the surface between the stretching and viscous terms in each cell. Advection transports the vorticity created by tilting near the surface downward. Figures 10a and $10 \mathrm{~b}$ also show that the vorticity budget is essentially closed over the conditionally averaged LC. However, perfect closure is not required; conditional averaging may result in a domain with "leaks" that are important to the vorticity budget. In these simulations, the 

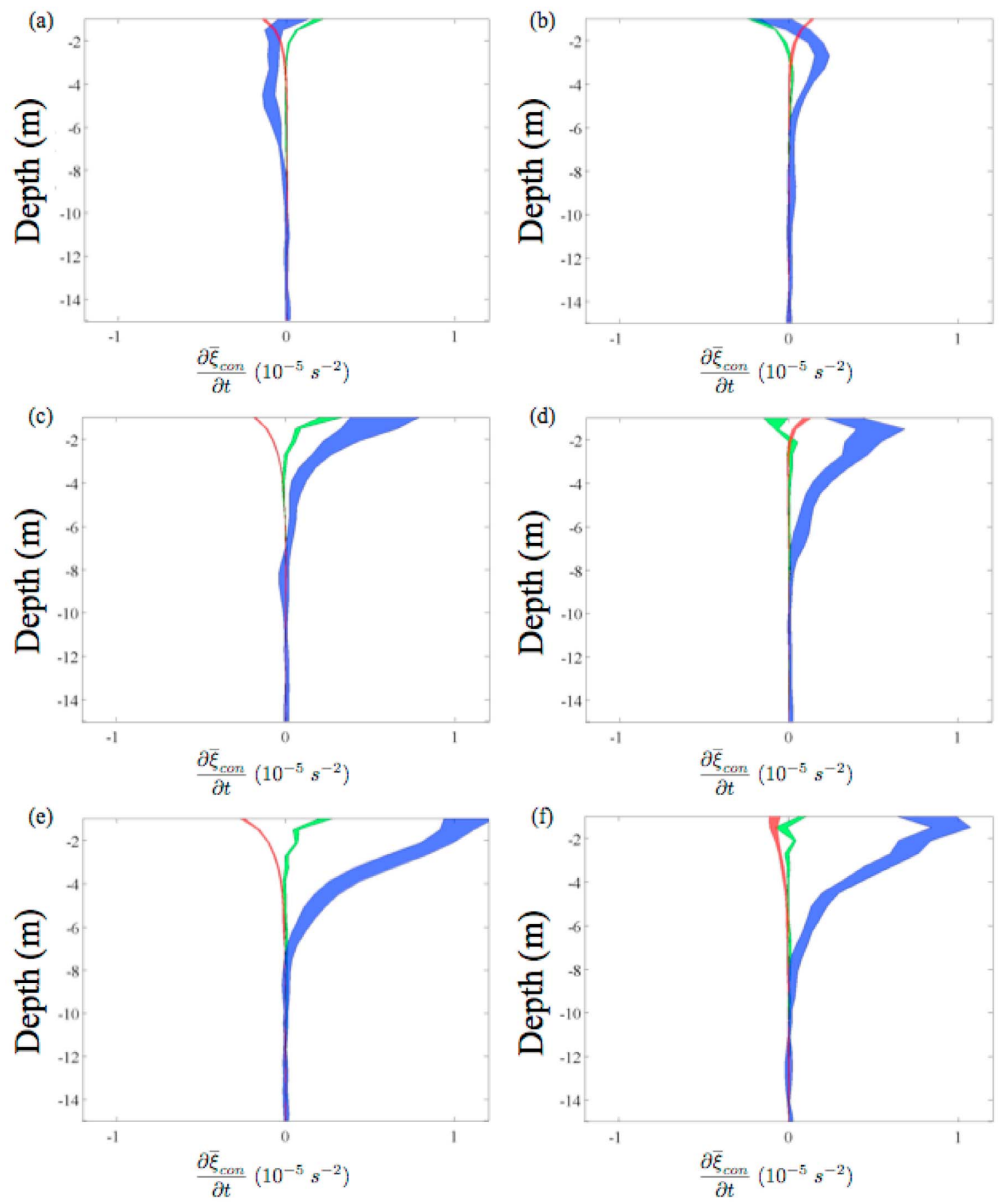

Figure 10. (a, c, and e) Conditionally averaged vorticity tendency terms in non-rotating cases averaged over the downwind side (blue left-hand vortex in Figure 9) of the average LC pair. (b, d, and f) The corresponding averages for the upwind half of the average LC pair (red right-hand vortex in Figure 9). The upwind and downwind averages include the midpoint of the domain and extend outward to where the axial vorticity $(\xi)$ falls to $10 \%$ of its maximum value. Figures $10 \mathrm{a}$ and $10 \mathrm{~b}$ are the aligned case, Figures $10 \mathrm{c}$ and $10 \mathrm{~d}$ are for $\theta_{w w}=45^{\circ}$, and Figures $10 \mathrm{e}$ and $10 \mathrm{f}$ are for $\theta_{w w}=90^{\circ}$. Blue is the advective tendency from (9), red is stretching from (9), and green is the viscous effect (calculated as a residual) given

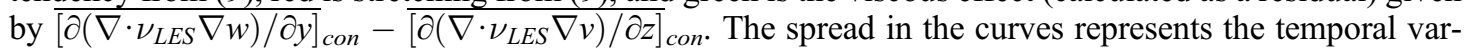
iation of the tendency terms over the final portion of the runs. 

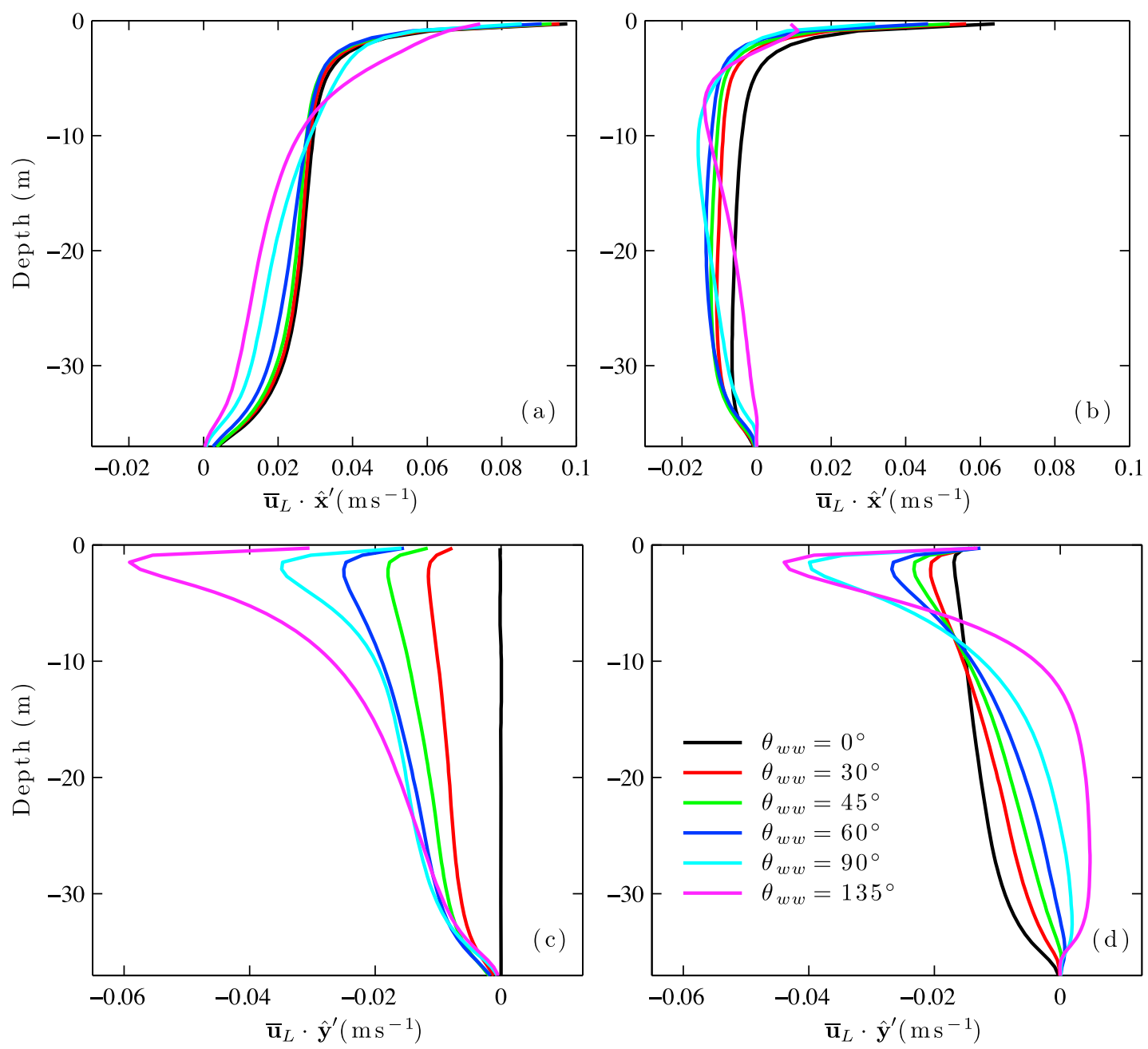

Figure 11. Profile of mean Lagrangian velocities rotated into the LC-aligned coordinate system with unit-length basis vectors $\left(\hat{\mathbf{x}}^{\prime}, \hat{\mathbf{y}}^{\prime}\right)$. The velocities (a) $\overline{\mathbf{u}}_{L} \cdot \hat{\mathbf{x}}^{\prime}$ without Coriolis, (b) $\overline{\mathbf{u}}_{L} \cdot \hat{\mathbf{x}}^{\prime}$ with Coriolis, (c) $\overline{\mathbf{u}}_{L} \cdot \hat{\mathbf{y}}^{\prime}$ without Coriolis, and (d) $\overline{\mathbf{u}}_{L} \cdot \hat{\mathbf{y}}^{\prime}$ with Coriolis are shown. Here $\overline{\mathbf{u}}_{L} \equiv \overline{\mathbf{u}}+\mathbf{u}_{s}$ is the mean Lagrangian velocity.

vorticity budget in the conditionally averaged LC is closed for the aligned case when $f=0 \mathrm{~s}^{-1}$ and $\theta_{w w}=0^{\circ}$, but not otherwise.

[52] As $\theta_{w w}$ increases, Figures $10 \mathrm{c}$ and $10 \mathrm{e}$ show that the magnitude of the stretching term near the surface in the downwind portion of the LC increases slightly and penetrates to greater depths. The changes in penetration depth of the viscous terms with $\theta_{w w}$ are similar to the changes in the stretching terms. The most dramatic change in Figures 10c and $10 \mathrm{e}$ occurs in the vorticity advection term; this term becomes increasingly positive as $\theta_{w w}$ increases, consistent with the decreasing negative vorticity of the downwind cell shown in Figure 9. Moreover, the cross-cell wind follows the direction of $\overline{\mathbf{u}} \cdot \hat{\mathbf{y}}^{\prime}$. This wind adds a frictional surface torque that magnifies the upwind cell vorticity and opposes the downwind cell vorticity. This surface frictional injection of vorticity further increases the asymmetry seen in Figure 9. As a result, a combination of viscous and advective effects from the cross-cell wind and Eulerian current accounts for the asymmetry in $\xi$ between the two cells shown in Figure 9 for $\theta_{w w}>0^{\circ}$.
[53] For the upwind cell $\left(y^{\prime}>0\right)$, Figure 10b shows that, for $\theta_{w w}=0^{\circ}$, the signs of the vortex diffusion and stretching/ tilting terms are opposite to those in the downwind cell (Figure 10a). Unlike the downwind cell, however, as $\theta_{w w}$ increases to $90^{\circ}$ (Figures $10 \mathrm{~d}$ and 10f), the stretching/tilting and diffusion terms are no longer in approximate balance in the upwind cell. In fact, these terms decrease in magnitude and change signs as $\theta_{w w}$ increases. At $\theta_{w w}=90^{\circ}$, the stretching/tilting terms have a negative contribution to the budget of $\xi$ in both the upwind and downwind cells. Consequently, in both cells tilting near the surface opposes advection. The general pattern of vorticity advection in the upwind cell is similar to that seen in the downwind cell. Note that the approximate balance in the aligned case (Figures 10a and 10b) is no longer seen in the non-aligned cases. The vorticity advection term is positive in both portions of the domain. This tendency continues to strengthen with larger values of $\theta_{w w}$.

[54] Variations in the advection of $\xi$ can be understood, in part, from the profiles of $\mathbf{u}_{L}$ in the along-cell and cross-cell directions shown in Figure 11. The cross-cell profiles in the 


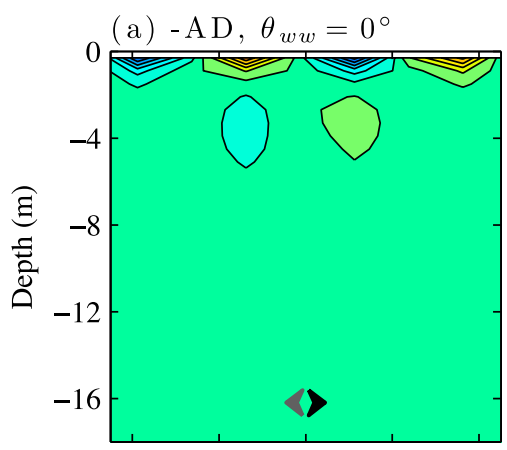

(d) TS, $\theta_{w w}=0^{\circ}$

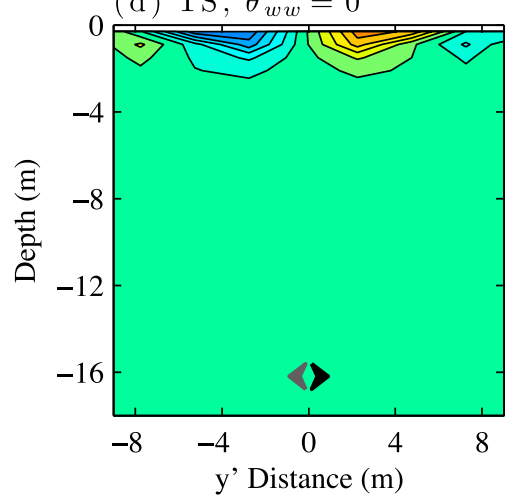

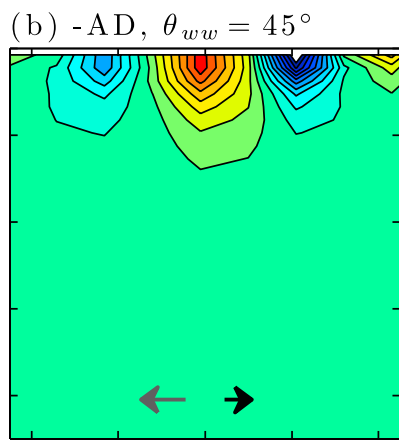

(e) $\mathrm{TS}, \theta_{w w}=45^{\circ}$

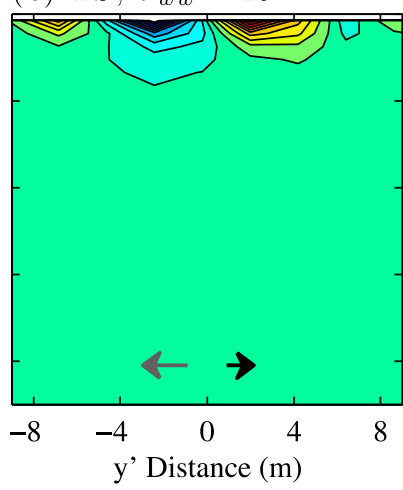

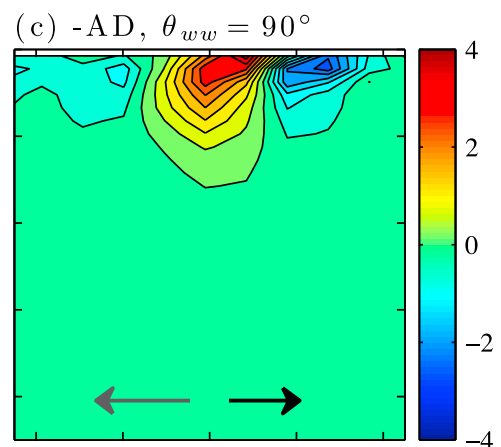

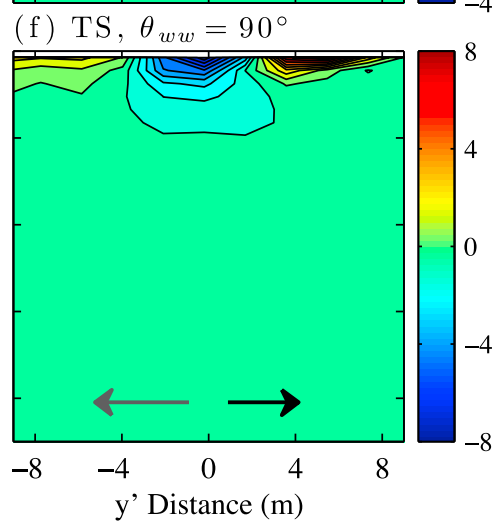

Figure 12. Cross-sections in the $\left(y^{\prime}, z\right)$ plane of conditionally-averaged negative advection $(-\mathrm{AD})$ and tilting/stretching (TS) budget terms in the evolution of $\xi$ (see (9)). (a-c) Negative advection terms are shown in non-rotating cases for $\theta_{w w}=0^{\circ}, 45^{\circ}$, and $90^{\circ}$, and $(\mathrm{d}-\mathrm{f})$ corresponding tilting/stretching terms are shown. The arrows at the bottom of the figures are as in Figure 9. Advection colorbars are scaled by $10^{-4}$ and tilting/stretching colorbars are scaled by $10^{-5}$.

non-rotating case (Figure 11c) become increasingly negative as $\theta_{w w}$ increases, consistent with an increasingly strong advection of $\xi$ from upwind to downwind cells. This tendency is somewhat weaker for the Coriolis cases, (Figure 11d), since here the cross-cell velocity is only weakly negative near the surface, and close to zero (or even positive) at all other depths. These differences for the Coriolis cases are due to the Ekman spiral.

[55] Note that, taken alone, we would not expect advection to increase $\xi$ in both the upwind and downwind cells. Since the budget terms in the upwind and downwind cells are obtained by averaging the terms over $y^{\prime}>0$ and $y^{\prime}<0$, respectively, a large positive advection near the middle of the conditionally-averaged domain (i.e., near $y^{\prime}=0$ ) would increase the advection in both cells. This can be seen from the two-dimensional cross-sections $\left(y^{\prime}-z\right.$ planes $)$ of the conditionally-averaged advection and tilting/stretching terms shown in Figure 12. In particular, the similarity between the downwind and upwind budgets for $\theta_{w w}=0^{\circ}$ in Figures 10a and 10b is now more obvious in Figures $12 \mathrm{a}$ and 12b. In Figure 12a,there are opposing lobes of positive and negative total advection in the upwind and downwind cells $\left(y^{\prime}>0\right.$ and $y^{\prime}<0$, respectively) that yield a near vorticity balance when summed over the LC. The same is true of the vortex tilting terms in Figure $12 \mathrm{~b}$ which, when summed over the domain, yield a total contribution to $\xi$ that is nearly zero (Figure 6).

[56] For $\theta_{w w}>0^{\circ}$, profiles of the advection terms in Figures 12c and 12e show substantial differences compared to the aligned case in Figure $12 \mathrm{a}$. For $\theta_{w w}=45^{\circ}$ and $90^{\circ}$, the approximate balance in Figure $12 \mathrm{a}$ is replaced by a strong positive tendency in the middle of the LC pair, while there are negative lobes further from the center (Figures 12c and 12e). Again, this pattern of advection in the misaligned cases is attributed to the advection of axial vorticity by the mean Eulerian velocity overcoming the Stokes drift velocity. The spatial structure and magnitude of the advective terms is consistent with Figures 10c and 10e.

[57] The vortex tilting/stretching terms in Figures $12 \mathrm{~d}$ and $12 \mathrm{f}$ are similar for the misaligned cases. Upon closer inspection, however, the strong negative lobe in the tilting/ stretching term shifts upwind to greater $y^{\prime}$ as $\theta_{w w}$ increases. This variation explains the switch in sign of the averaged tilting term seen in Figure 10f. With a strong positive advective tendency in the center of the domain, the tilting terms shift in the upwind direction and combine with the diffusion term to compensate advection.

[58] The preceding analysis has been based primarily on results in the non-rotating cases. For the simulations with rotation, however, the structure of the vorticity budgets vary slightly. Figure 13 shows that the domain averaged budgets of $\xi$ for $\theta_{w w}=0^{\circ}$ with Coriolis forcing are different than the corresponding non-rotating budgets in Figure 6a. In particular, Figure 13 suggests that the advection, tilting/stretching, and diffusion terms all have nonzero mean profiles in the rotating case, while all three averages were approximately zero in the non-rotating case. The shaded regions in Figure 6a encompass half of the inertial oscillation (from the 


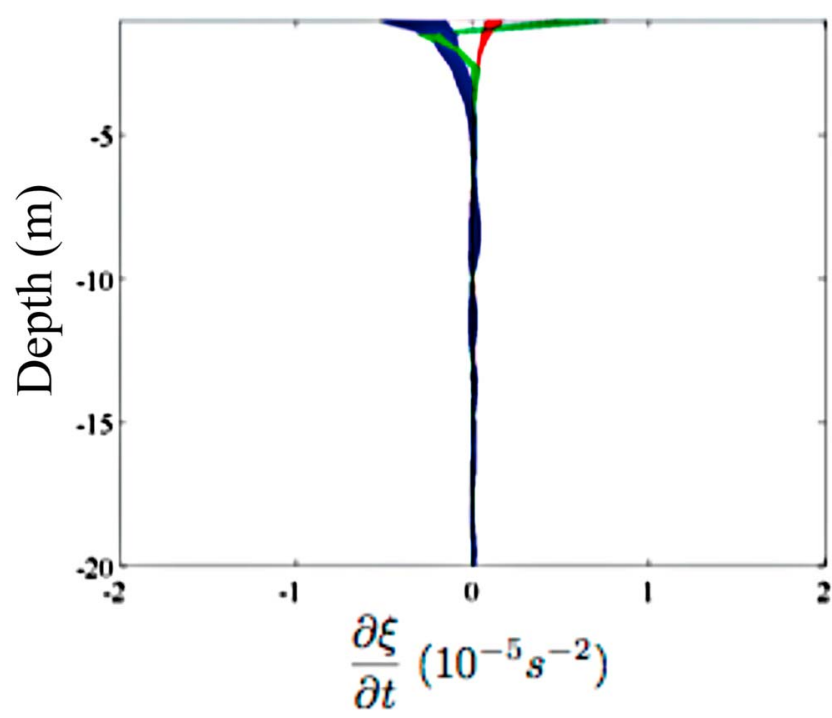

Figure 13. Select forcing terms for the runs with Coriolis forcing. Domain averaged vorticity forcing for the aligned case. Blue is the advective tendency from (9), red is stretching from (9), and green is the viscous effect (calculated as a residual) given by $\overline{\left[\partial\left(\nabla \cdot \nu_{L E S} \nabla w\right) / \partial y\right]}$ con $\left.\overline{\left[\partial\left(\nabla \cdot \nu_{L E S} \nabla v\right) / \partial z\right.}\right]_{c o n}$. The spread in the curves represents the temporal variation of the tendency terms over the final portion of the runs. minimum $v$ velocity magnitude to the maximum magnitude). For the rotating cases, a non-negligible tendency term results from inertial oscillations. What is most intriguing about the rotating, aligned case is the strong advective forcing for $\xi$ not seen in the non-rotating, aligned case. Figure 11 shows that the magnitude of the cross-cell Lagrangian velocity in the aligned, rotating case is similar to the cross-cell advection in the misaligned, non-rotating cases. Furthermore, the LC asymmetry in Figure 13 is similar to that from the non-rotating case with $\theta_{w w}=30^{\circ}$ (not shown). Thus, cross-cell advection, by misaligned waves and wind or Ekman spiral, is a primary cause of the asymmetry of the LC.

[59] The horizontal structure of the stretching tendency term (Figures $14 \mathrm{c}$ and $14 \mathrm{f}$ ) is similar in the rotating simulations, indicating that $(\zeta+f) \frac{\partial \mathbf{u}_{L}}{\partial z} \approx \zeta \frac{\partial \mathbf{u}_{L}}{\partial z}$. Thus, the effective Rossby number of the LC is large. When the misalignment is increased to $60^{\circ}$ (Figures 14e and 14f), the magnitudes of the stretching tendency terms again shift upwind, as in the non-rotating cases.

\section{Effects of Forcing Parameter Variations}

[60] In addition to the two basic sets of simulations discussed to this point (i.e., the rotating and non-rotating simulations using the parameters summarized in Table 1), additional simulations have also been carried out to determine the robustness and representativeness of the results when the forcing parameters are varied. Two important variations in the forcing are discussed in this section: the
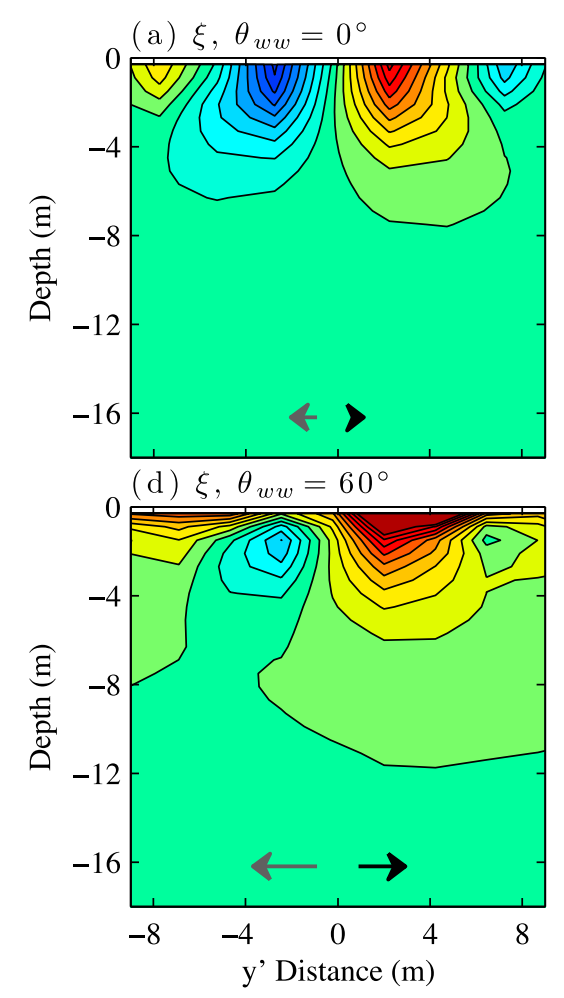
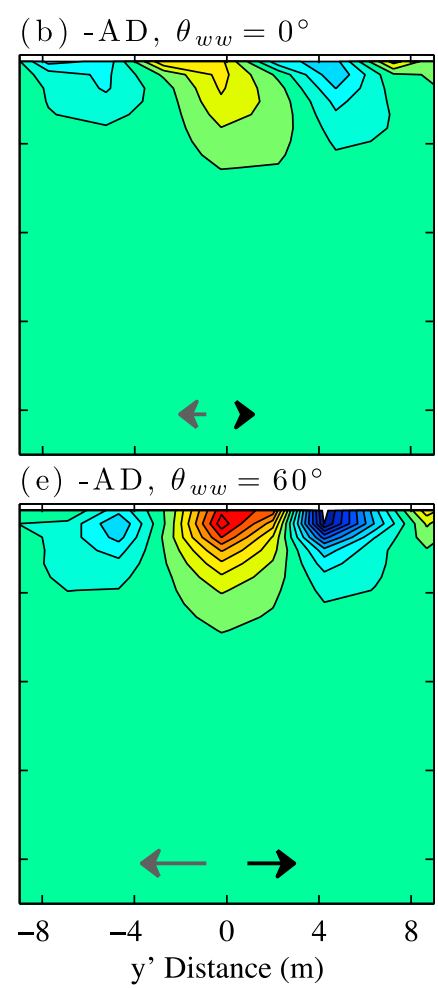
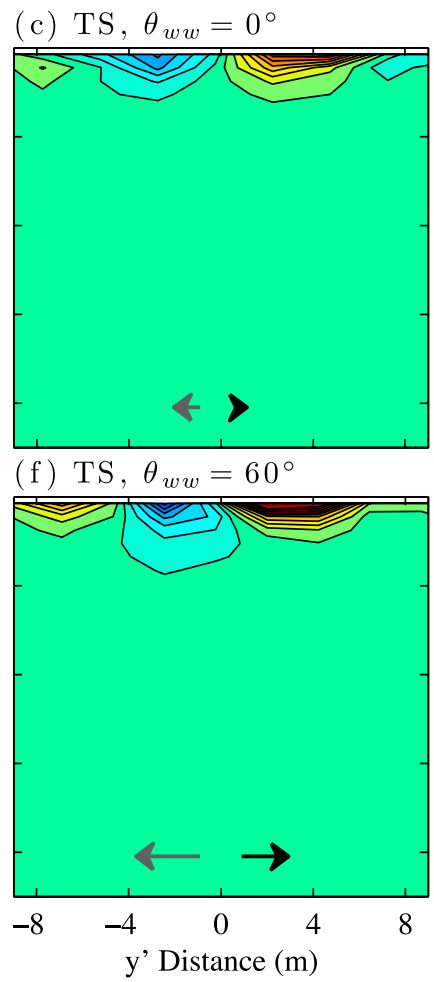

Figure 14. Conditionally averaged horizontal structure of $\xi$, advection $(-\mathrm{AD})$, and tilting/stretching (TS) for rotating cases with $\theta_{w w}=0^{\circ}$ and $\theta_{w w}=60^{\circ}$. The (a-c) $\xi,-\mathrm{AD}$, and TS for $\theta_{w w}=0^{\circ}$ and (d-f) $\xi$, $-\mathrm{AD}$, and TS for $\theta_{w w}=60^{\circ}$ are shown. The arrows at the bottom of the figures are as in Figure 9 and the colorbars are the same as in Figures 9 and 12. 


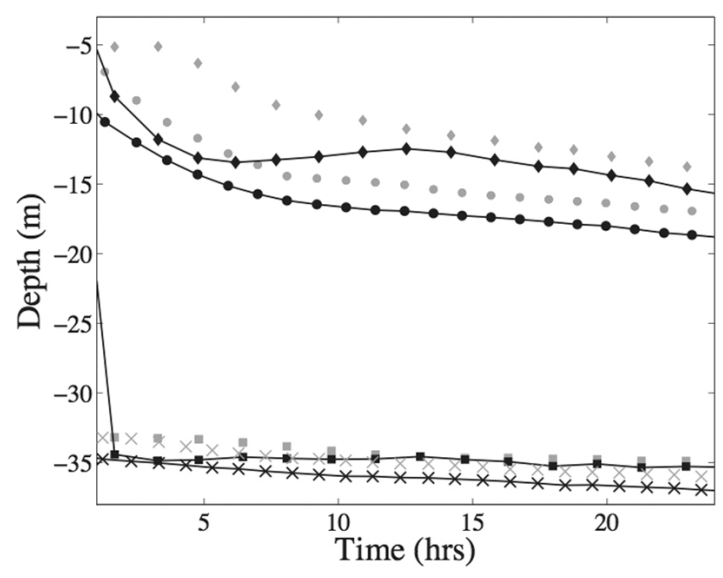

Figure 15. Depth where $R i \geq 0.3$, which is taken to indicate the mixing depth (gray symbols) and mixed layer depth (black lines with symbols) in time. Cases with an initial mixed layer are shown by crosses (with Stokes drift) and by squares (without Stokes drift). Cases without an initial mixed layer are shown by circles (with Stokes drift) and by diamonds (without Stokes drift).

inclusion of wind waves in the Stokes drift profile and the removal of the mixed layer from the initial conditions.

[61] The simulations with aligned wind waves are carried out for both rotating and non-rotating cases and for $\theta_{w w}=$ $0^{\circ}-135^{\circ}$. The total Stokes drift for the wind-wave simulations is thus the superposition of young wind waves and older, misaligned waves based on the preconditioning winds. We find that the inclusion of wind waves does not substantially alter the conclusions from the preceding sections, for two reasons. First, the angle predictions discussed in section 5 are independent of how the Stokes drift profile is composed. That is, once the Stokes drift is established, regardless of the type and number of superimposed wavefields, it can then be used to construct the various angle predictions in section 5 . Second, the frequency of the wind waves is an order of magnitude higher than that of the misaligned waves. Consequently, when the wind-wave spectrum is integrated, the resulting contribution to the total Stokes drift is very small (at the surface, $\left|\mathbf{u}_{s}(0)\right| \approx 10^{-8} \mathrm{~ms}^{-1}$ for the wind-waves and $\left|\mathbf{u}_{s}(0)\right|=0.06313 \mathrm{~ms}^{-1}$ for the misaligned waves). Situations where the Stokes drift results from two or more equally-matched wavefields in different directions are conceivable, but the parameter space of such scenarios is too large to explore in depth here.

[62] Simulations with no mixed layer in the initial stratification, but with all other parameters in Table 1 unchanged, were also run for the rotating case. The motivation for these simulations is that in the basic set of simulations with an initial mixed layer, the LC do not penetrate strongly through the entire mixed layer, and entrainment is therefore weak. The along-cell vorticity, $\xi$, is only significant in the upper few meters (as shown in section 6), and while significant vertical velocities penetrate below the upper few meters, VKE and subgrid TKE are weak near the mixed layer base. Many previous simulations have deeper penetration, but use different Stokes drift forcing [McWilliams et al., 1997; Noh et al., 2004; Sullivan et al., 2004; Li et al., 2005; Grant and Belcher, 2009]. Nonetheless, LT does extend below the obvious coherent structures to result in an increased entrainment rate relative to a pure ST simulation. Figure 15 shows the mixed layer depth and the mixing depth (or boundary layer depth) where the critical Richardson number $(0.3)$ is reached. The basic cases with an initial mixed layer show small differences between the LT and ST cases. The cases without an initial mixed layer show profound enhanced entrainment when Stokes drift forcing is included. The increased entrainment is consistent with increased mixing depth, as diagnosed by a critical Richardson number criterion. Thus, mixing and entrainment due to LT are evident in the no initial mixed layer cases, even though near the mixed layer base no obvious coherent LC structures appear in the conditional averaging (not shown).

[63] In misaligned cases with no initial mixed layer, the entrainment rate and mixing depth decrease with increasing $\theta_{w w}$. This is consistent with the reduction in VKE as $\theta_{w w}$ increases for the initial mixed layer simulations in Figure 3. In the no initial mixed layer cases, the final mixed layer depth depends on $\theta_{w w}$; the mixed layer is deepest for $\theta_{w w}=0^{\circ}$, of intermediate depth for $\theta_{w w}=90^{\circ}$, and shallowest when the Stokes drift is not present (i.e., shear only). The entrainment at the mixed layer base in the no initial mixed layer cases is thus consistent with the decrease in $\overline{w^{\prime 2}}$ with $\theta_{w w}$ shown in Figure 3.

[64] The no initial mixed layer cases allow us to elaborate on the choice of averaging depth, $D_{l}$, used in section 5 as compared to mixed layer, mixing, and wave-based depths. Cases with no initial mixed layer strongly suggest that twice the $e$-folding depth $(3 \mathrm{~m})$ of the peak wave, which is $5.1 \mathrm{~m}$, not 20\% of the mixed layer depth, should be used for the depth $D_{l}$ in the angle predictions and TKE calculations. Those angles are based on the mixing depth, or depth over which the TKE production occurs. However, as shown in Figure 15, the mixed layer depths may vary widely between these cases while the mixing depths are quite similar. Regardless of mixed layer depth, the layer that contains $95 \%$ of the TKE production is roughly equivalent to a layer that contains $90 \%$ of the peak wave Stokes shear. In more realistic scenarios, restratification mechanisms [Fox-Kemper et al., 2008, 2011] (e.g., solar heating or mixed layer eddies) would reduce the mixed layer depth toward the Langmuir or other boundary layer mixing depth where turbulence is strong. Thus, one may expect better agreement between $D_{L}=0.2 H_{M L}$ and twice the $e$-folding depth of the wave spectrum in realistic situations than in the transient, nonequilibrium scenarios studied here.

\section{Langmuir Number Scaling}

[65] In prior studies of aligned winds and waves [e.g., McWilliams et al., 1997; McWilliams and Sullivan, 2000], the turbulent Langmuir number, $L a_{t}$, was given as the ratio of the surface wind stress, $u_{*}$, to the surface Stokes velocity in the wind direction, $u_{s}(0)$. For misaligned winds and waves, however, such a straightforward definition of $L a_{t}$ is no longer accurate, since the surface wind stress and Stokes velocity have components both parallel and perpendicular to LC. Following Belcher et al. (unpublished manuscript, 2011), among others, the Langmuir number may be defined more generally as the ratio of the TKE production by 
Eulerian and Stokes shears (although McWilliams et al. [1997] argue for turbulent Langmuir number as a ratio of terms in the momentum equation, not the energy equation), which is written as

$$
L a_{T K E}^{2}=\frac{\overline{\mathbf{u}^{\prime} w^{\prime}} \cdot(\partial \overline{\mathbf{u}} / \partial z)}{\overline{\mathbf{u}^{\prime} w^{\prime}} \cdot\left(\partial \mathbf{u}_{s} / \partial z\right)} .
$$

Based on the discussion of the time-dependent TKE budget in section 4 (see also Figure 5), it is emphasized that the Langmuir number is related to the production ratio (22) during the onset of $L T$. Here we generalize $L a_{t}$ to the misaligned cases by defining a new turbulent Langmuir number, denoted $L a_{\text {proj }}$, as the ratio of the wind stress and surface Stokes drift velocity projected into the LC direction. The resulting Langmuir number is then written as

$$
L a_{p r o j}^{2} \equiv \frac{\left|\mathbf{u}_{*}\right| \cos \left(\alpha_{\mathcal{L}}\right)}{\left|\mathbf{u}_{s}(0)\right| \cos \left(\theta_{w w}-\alpha_{\mathcal{L}}\right)},
$$

where we have used $\alpha_{\mathcal{L}}$ for the LC direction due to the connection between (22) and the TKE budget in section 4 . This generalized form for $L a_{p r o j}$ in (23) reduces to $L a_{t}$ when $\theta_{w w}=0^{\circ}$.

[66] Since many of the simulations discussed to this point give $\alpha_{\mathcal{L}} \approx 0.5 \theta_{w w}$ (see Figure 7$), L a_{p r o j}^{2} \approx L a_{t}^{2}$ from (23) and we are unable to determine how misalignment influences LT mixing strength over ST. To examine the validity of (23) over a broader range of conditions, additional simulations with varying Stokes drift magnitude were performed (as summarized in Table 2). In these simulations, the Stokes drift magnitude was varied by equilibrating the Donelan et al. [1985] spectra using a range of different preconditioning wind speeds (given in Table 2). Despite these different preconditioning wind speeds, however, the simulation wind speed is maintained at $U_{10}=5.75 \mathrm{~ms}^{-1}$ for all cases. Five different values of the preconditioning wind speed were used, and Table 2 shows that the equivalent values of $L a_{t}$ vary by nearly a factor of 3 . For each value of $L a_{t}$, three different misalignments were examined: $\theta_{w w}=0^{\circ}, 30^{\circ}$, and $60^{\circ}$. The value of $L a_{\text {proj }}$ from (23) varies by roughly $15 \%$ for these values of $\theta_{w w}$.

[67] Previous studies [Li et al., 2005; Harcourt and D'Asaro, 2008] have indicated that the normalized VKE shown in Figure 3 should scale with $L a_{t}$. Here we examine the functional form given by

$$
\frac{\left\langle\overline{w^{\prime 2}}\right\rangle_{H_{M L}}}{\left[u_{*} \cos \left(\alpha_{\mathcal{L}}\right)\right]^{2}}=0.6\left[1.0+\left(c_{1} L a_{x}\right)^{-2}+\left(c_{2} L a_{x}\right)^{-4}\right],
$$

where the left-hand side is obtained by averaging the normalized VKE profiles in Figure 3 over the full mixed layer depth (using the averaging definition in (16) with $D_{l}=H_{M L}$ ). The coefficients $c_{1}$ and $c_{2}$ in (24) are obtained from the simulation data using a non-linear least squares routine, and the leading coefficient on the right-hand side is obtained from the reanalysis of $\mathrm{Li}$ et al. [2005] by Harcourt and D'Asaro [2008]. This value was determined using simulations that only include shear turbulence $\left(L a_{t} \rightarrow \infty\right)$ and that do not include Coriolis forcing, giving $\overline{w^{\prime 2}} / u_{*}^{2}=0.64$ [ Li et al.,
2005]. In our shear-only simulation, we find $\overline{w^{\prime 2}} / u_{*}^{2}=0.55$, which is still in relatively good agreement with the leading coefficient in (24); in the rotating cases with $f=10^{-4} \mathrm{~s}^{-1}$, this coefficient decreases to 0.43 .

[68] In (24), $L a_{x}$ represents one of three possible definitions of Langmuir number: either $L a_{t}=u_{*} /\left|\mathbf{u}_{s}(0)\right|, L a_{\text {prof }}$ from (23), or the Langmuir number from equation (32) of Harcourt and D'Asaro [2008]. The Langmuir number from Harcourt and D'Asaro [2008], which we denote $L a_{S L}$, is a projected generalization of the surface layer averaged Langmuir number, which covers both exponential and empirical wave spectra. Note that we approximate $L a_{S L}$ based on the resolved Stokes shear, rather than integration of an analytic spectrum, which is likely to degrade our results versus those of Harcourt and D'Asaro [2008]. Precise agreement is not the goal here; rather, the goal is merely to show that the projection of $L a_{S L}$ is an improvement over ignoring misalignment. Once again, for the misaligned cases, $\mathbf{u} *$ and $\mathbf{u}_{s}$ are both projected into the LC direction. Scaling results from the simulations are shown for each of these three definitions of $L a_{x}$ in Figure 16. For $L a_{x}=L a_{t}$ in Figure 16a, the values of $c_{1}$ and $c_{2}$ in (24) were found to be $c_{1}=3.1$ and $c_{2}=5.7$. Figure $16 \mathrm{a}$ shows that the resulting fit to our simulation data is slightly different than the Harcourt and D'Asaro [2008] fit of the Li et al. [2005] data, but if the misaligned cases (i.e., $\theta_{w w} \neq 0^{\circ}$ ) are neglected, then there is close agreement. If the $95 \%$ confidence bounds are taken into account, which are [2.3, 4] and [3.4, 6] for $c_{1}$ and $c_{2}$, respectively, the fits agree with Harcourt and D'Asaro [2008], including the misaligned cases.

[69] Although the scatter of the simulation results around the fit line is not large in Figure 16a, it is significant and systematically biased. In Figure $16 \mathrm{~b}, L a_{\text {proj }}$ from (23) is used instead of $L a_{t}$ in (24), and the resulting fit matches the simulation data more closely, with a smaller quartic coefficient. Based on the size of the uncertainty in $\alpha_{\mathcal{L}}$, all of the simulation values lie on the curve, and the fit cannot be improved further. Considering the projections of $\mathbf{u} *$ and $\mathbf{u}_{s}$ into the LC direction moves the predicted mixing strength onto the fit line, suggesting that $L a_{\text {proj }}$ is the appropriate non-dimensional number to consider for misaligned wind and waves.

[70] Finally, fits using $L a_{S L}$ from Harcourt and D'Asaro [2008] are shown in Figure 16c. Once again, projecting $\mathbf{u}_{*}$ and $\mathbf{u}_{s}$ into the LC direction improves the fit by shifting the results onto the fit line. However, some of the values in Figure 16c are still statistically different from the fit curve. For $L a_{S L} \leq 1$, Harcourt and D'Asaro [2008] suggest a power law scaling of the form

$$
\frac{\left\langle\overline{w^{\prime 2}}\right\rangle_{H_{M L}}}{u_{*}^{2}}=c_{3}+c_{4} L a_{S L}^{-4 / 3},
$$

where $c_{3}=0.398$ and $c_{4}=0.48$, as determined using LES results. When all of the aligned and misaligned cases are considered in the present simulations, we obtain $c_{3}=0.063$ and $c_{4}=0.68$, with $95 \%$ confidence bounds of $[-0.29,0.41]$ and $[0.57,0.80]$, respectively. The scaling from Harcourt and D'Asaro [2008] with their coefficients is shown as the thin line in Figure $16 \mathrm{c}$ and our coefficients generate the 
dashed line. The results of Harcourt and D'Asaro [2008] fall within our confidence bounds for $c_{3}$, but not for $c_{4}$. However, uncertainty in $\alpha_{\mathcal{L}}$ and the fact that we use a subtly different definition of $L a_{S L}$ (resolved Stokes values instead of a spectral integral) likely explain why our value of

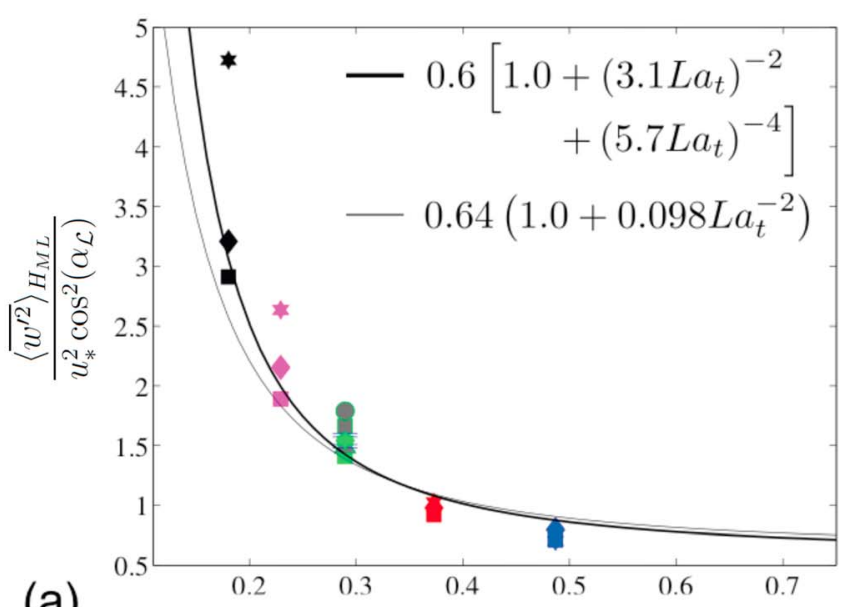

(a)

$$
L a_{t} \equiv\left(\frac{\left|u_{*}\right|}{\left|u_{s}(0)\right|}\right)^{1 / 2}
$$

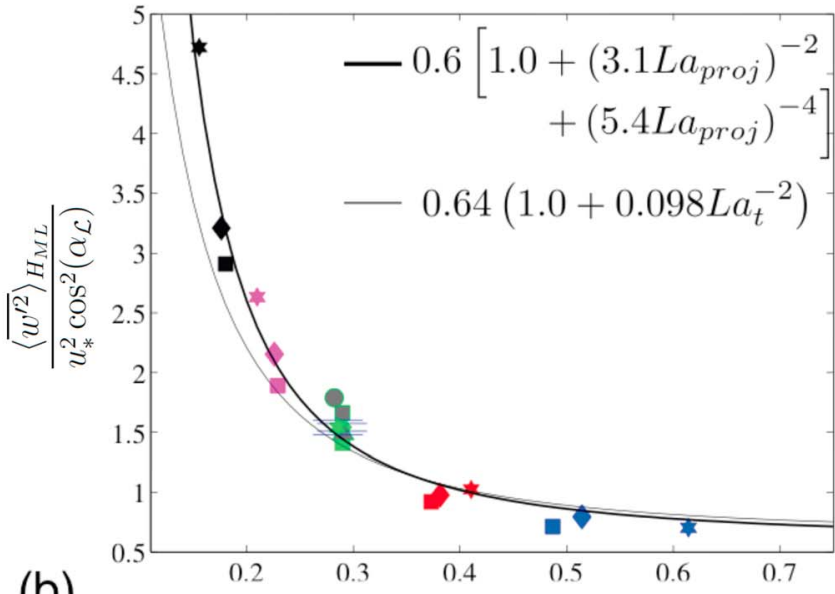

(b)

$$
L a_{\text {proj }} \equiv\left(\frac{\left|u_{*}\right| \cos \left(\alpha_{\mathcal{L}}\right)}{\left|u_{s}(0)\right| \cos \left(\theta_{w w}-\alpha_{\mathcal{L}}\right)}\right)^{1 / 2}
$$

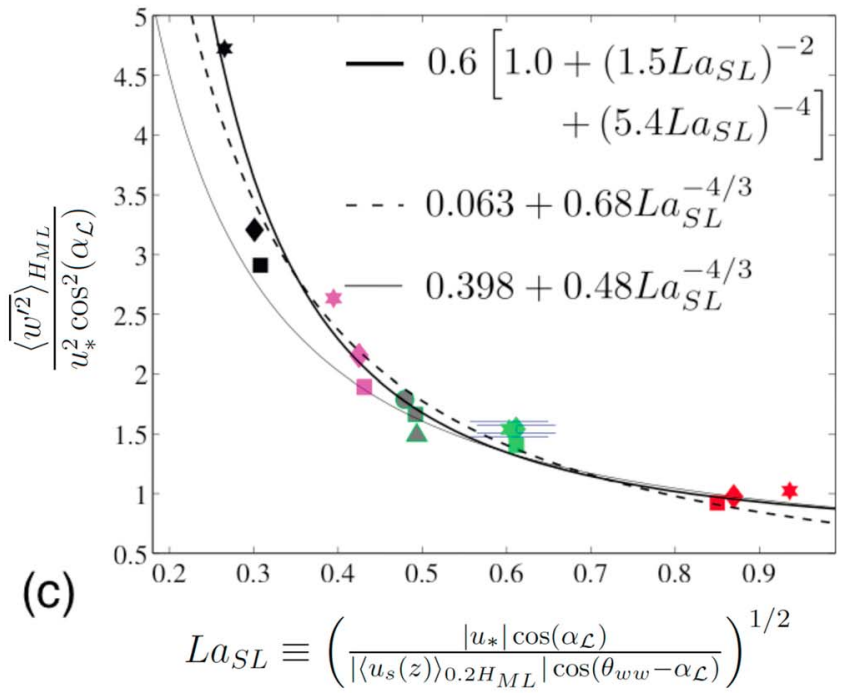

$c_{4}$ differs from Harcourt and D'Asaro [2008]. The quartic functional form used in Figures 16a and $16 \mathrm{~b}$ is also shown in Figure 16c with nonlinear fit coefficients for reference. Some additional simulations suggested to us (R. R. Harcourt, personal communication, 2011), where the depth-profile of Stokes drift is varied, do confirm that realistic spectra are better quantified by a turbulent Langmuir number that has sub-surface Stokes drift information (such as $L a_{S L}$ ) than a surface-only version (such as $L a_{t}$ ). Thus, the conclusion of Harcourt and D'Asaro [2008] is confirmed despite the differences in fit coefficients for our simulations ascribed to the model setup above.

\section{Discussion and Conclusions}

[71] LES have been performed for wave spectra away from full-development with the Stokes drift rotated and changed in magnitude relative to a fixed wind. Simulations with and without Coriolis forcing were conducted. Predictions of the angle of the LC axial direction relative to the wind stress, $\alpha$, were compared to the results. The resulting angle is not strongly affected by the presence or absence of the Coriolis force. In these simulations, the optimal prediction that does not require LES results is derived in section 5 using the law of the wall and is given by

$$
\tan \left(\alpha_{L O W}\right)=\frac{\left\langle\partial v_{s} / \partial z\right\rangle_{D_{l}}}{\frac{u_{*}}{\kappa\left|z_{1}-D_{l}\right|} \ln \left(\left|D_{l} / z_{1}\right|\right)+\left\langle\partial u_{s} / \partial z\right\rangle_{D_{l}}} .
$$

This formulation is sensitive to the chosen depth over which the shear is integrated, $D_{l}$, and the shallowest depth where the law of the wall is thought to apply $\left(z_{1}\right)$. Using $D_{l}=H_{M L}$, with $z_{1}$ as the first LES grid point, was found here to be optimal, but using $D_{l}=0.2 H_{M L}$, as suggested by Harcourt and D'Asaro [2008], resulted in little difference. In the real ocean, there is no first grid point, but a description of the depth where the Stokes drift and law of the wall might first be applied. Traditionally, the law of the wall applies just outside of the viscous sublayer, but the Stokes drift also depends on asymptotic assumptions regarding surface waves. Thus, a depth where one is clearly below the viscous sublayer and where the Stokes drift is likely to apply, such as a multiple of the significant wave height, would be a reasonable choice [Thorpe, 2007].

[72] In the present analysis $D_{l}$ was sometimes chosen as $0.2 H_{M L}$, which was found to be nearly the depth where $95 \%$ of the Stokes shear production occurred in most of our simulations. However, in simulations where the mixed layer depth was initially zero, the mixed layer rapidly deepened due to the Langmuir turbulence. Analysis of these simulations revealed that twice the $e$-folding depth of the peak

Figure 16. Scaling of normalized mixed layer averaged $\overline{w^{\prime 2}}$ with three definitions of Langmuir number (see Table 2). The curves are fit with a non-linear least squares algorithm with a functional fit given by $\frac{\left\langle\overline{w^{\prime 2}}\right\rangle_{H_{M L}}}{\left(u * \cos \left(\alpha_{\mathcal{L}}\right)\right)^{2}}=0.6\left(1+c_{1} L a_{x}^{-2}+\right.$ $\left.c_{2} L a_{x}^{-4}\right)$. In this fit $L a_{x}$ represents one of three possible definitions: (a) $L a_{t}^{2} \equiv \frac{\left|u_{*}\right|}{\left|u_{S}(0)\right|}$, (b) $L a_{\text {proj }}^{2} \equiv \frac{\left|u_{*}\right| \cos \left(\alpha_{\mathcal{L}}\right)}{\left|u_{s}(0)\right| \cos \left(\theta_{w w}-\alpha_{\mathcal{L}}\right)}$, and (c) $L a_{S L}^{2}$. 


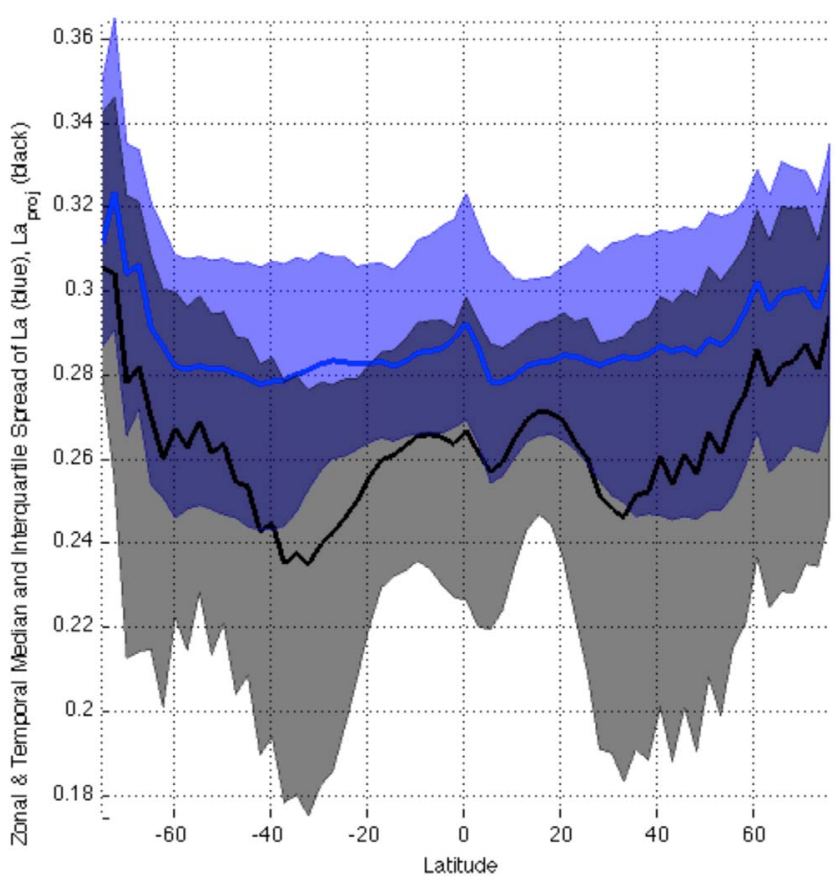

Figure 17. Temporal and zonal median and interquartile range of $L a_{t}$ and $L a_{\text {proj }}$ for a realistic simulation of 19942002 using Wave Watch III.

wave in the empirical spectrum may be a more robust estimate of $D_{l}$ when the mixed layer depth is evolving. In the equilibrated mixed layer simulations carried out here, these two estimates were in agreement.

[73] For small misalignments $\left(\theta_{w w} \leq 30^{\circ}\right)$, most reasonable predictions of $\alpha$ (given in section 5) agree with each other and the LES. As misalignment increases, only the predictions based on LES statistics and $\alpha_{L O W}$ remained accurate. Some estimates of the orientation and strength of LC have serious drawbacks. The presumption that $\mathrm{LC}$ requires a portion of the Stokes drift to project onto the wind stress (i.e., $\theta_{w w}<90^{\circ}$ ) does not hold, since a coherent LC structure is seen when $\theta_{w w}=90^{\circ}$, and a weak LC-like structure is seen at $\theta_{w w}=135^{\circ}$.

[74] For Stokes drift that differs in direction but not magnitude from the fully-developed wavefield for a given wind ( $L a_{t} \approx 0.3$ ) the orientation of LC is nearly $\alpha \approx 0.5 \theta_{w w}$. When $\alpha \approx 0.5 \theta_{w w}, L a_{p r o j}$ is equal to $L a_{t}$.

[75] Scaling relationships for the strength of Langmuir mixing have been derived. The strength of LC mixing for misaligned wind and waves $\left(\theta_{w w} \neq 0^{\circ}\right)$ follow previously derived scalings under two conditions. First, $\overline{w^{\prime 2}}$ must be normalized by the projection of the surface stress into the LC direction instead of the total magnitude. Second, the turbulent Langmuir number must be reinterpreted as defined in (23). Projecting vector quantities into the axial direction of the LC collapses the LT statistics, mixing rate, and entrainment onto nearly the aligned result for a wide range of parameters. This procedure is summarized by the following:

$$
\begin{gathered}
\overline{w^{\prime 2}} \propto u_{*}^{2} \rightarrow \overline{w^{\prime 2}} \propto u_{*}^{2} \cos ^{2}(\alpha) \\
L a_{t}^{2}=\frac{\left|u_{*}\right|}{\left|u_{s}\right|} \rightarrow L a_{p r o j}^{2}=\frac{\left|u_{*}\right| \cos (\alpha)}{\left|u_{s}\right| \cos \left(\theta_{w w}-\alpha\right)} .
\end{gathered}
$$

A suitable estimate for $\alpha$ that does not require LES results is $\alpha \approx \alpha_{\text {LOW. }}$.

[76] The results of this generalization and the LES results from sections 3 and 5 may be combined to give a prediction for the maximum mixing due to LT based solely upon observed values of $u_{*}, u_{s}$, and $\theta_{w w}$, grouped nondimensionally when possible. If it is assumed that $\left\langle\partial u_{s} / \partial z\right\rangle_{D_{l}}=$ $u_{s}(0)-u_{s}\left(D_{l}\right) \approx u_{s}(0)$, then

$$
\frac{\left\langle\overline{w^{\prime 2}}\right\rangle_{H_{M L L}}}{u_{*}^{2}}=0.6 \cos ^{2}\left(\alpha_{L O W}\right)\left[1.0+\left(3.1 L a_{p r o j}\right)^{-2}+\left(5.4 L a_{p r o j}\right)^{-4}\right] \text {, }
$$

$$
\begin{gathered}
L a_{p r o j}^{2}=\frac{\left|u_{*}\right| \cos \left(\alpha_{L O W}\right)}{\left|u_{s}\right| \cos \left(\theta_{w w}-\alpha_{L O W}\right)}, \\
\alpha_{L O W} \approx \tan ^{-1}\left[\frac{\sin \left(\theta_{w w}\right)}{\frac{u_{*}}{u_{s}(0) \kappa} \ln \left(\left|H_{M L} / z_{1}\right|\right)+\cos \left(\theta_{w w}\right)}\right] .
\end{gathered}
$$

Results using different initial mixed layer depths indicate that care is needed when averaging over the "surface layer" as suggested by Harcourt and D'Asaro [2008]. The $e$-folding depth of the peak wave is shown to be superior hereto a fraction of the mixed layer depth as an estimator of the depth of wave production. If a boundary layer mixing depth is known from LES or an ocean mixing parameterization, it is likely to be a fair estimator for $L a_{S L}$. However, if only wave data or a wave model is available, the mixed layer depth may not be a good replacement for the "surface layer", although restratification mechanisms not included here tend to make the distinctions between mixed layer and mixing layer much smaller.

[77] The prediction (29) converges to $\alpha_{L O W}=\theta_{w w}$ in the case of strong Stokes drift and $\alpha_{L O W}=0^{\circ}$ in the case of strong winds. Likewise, when $\theta_{w w}=0^{\circ}$ the scalings agree with previous results for aligned waves and wind [Harcourt and D'Asaro, 2008].

[78] A useful set of bounds on the behavior of (29) can be found by considering the strong wind, strong wave, and balanced waves and winds. It begins from an identity that holds for all $\theta$ with a magnitude smaller than $\pi$

$$
\frac{\theta}{2}=\tan ^{-1} \frac{\sin \theta}{\cos \theta+1} \text {. }
$$

The bounds are based on this identity together with (26), and are

$$
\begin{gathered}
\text { If } L a_{t}^{2} \geq \frac{\kappa}{\ln \left(\frac{h}{z_{1}}\right)}, \quad \frac{L a_{t}^{2}}{\cos \left(\theta_{w w}\right)} \geq L a_{p r o j}^{2} \geq L a_{t}^{2}, \\
\text { Else if } L a_{t}^{2}=\frac{\kappa}{\ln \left(\frac{h}{z_{1}}\right)}, \quad L a_{t}^{2}=L a_{p r o j}^{2}, \\
\text { Else if } L a_{t}^{2} \leq \frac{\kappa}{\ln \left(\frac{h}{z_{1}}\right)}, \quad L a_{t}^{2} \cos \left(\theta_{w w}\right) \leq L a_{p r o j}^{2} \leq L a_{t}^{2} .
\end{gathered}
$$

Thus, for small $L a_{t}^{2}, L a_{p r o j}^{2}$ will be smaller than $L a_{t}^{2}$ by up to $\cos \left(\theta_{w w}\right)$. For large $L a_{t}^{2}, L a_{p r o j}^{2}$ may be larger by as much as a factor of $1 / \cos \left(\theta_{w w}\right)$. The point when $L a_{t}^{2}=L a_{p r o j}^{2}$, regardless 
of $\theta_{w w}$, is when the middle equality holds, which is approximately $10\left|\mathbf{u}_{*}\right|=\left|\mathbf{u}_{s}(0)\right|$.

[79] To appreciate the magnitude of the preceding rescalings, Figure 17 compares $L a$ and $L a_{\text {proj }}$ in a realistic 1994 2002 hindcast using Wave Watch III (details given by Webb and Fox-Kemper [2011]). L $a_{\text {proj }}$ is calculated with (28)-(29) using the density-criterion mixed layer depth $\left(\Delta \rho=0.03 \mathrm{~kg} \mathrm{~m}^{-3}\right)$ for $h$ from the mixed layer depth climatology of de Boyer Montégut et al. [2004] updated to include ARGO float data to September 2008. The onset depth of the law of the wall, $z_{1}$, is taken as four times the significant wave height [Thorpe, 2007]. The Stokes drift is calculated as $0.8 \mathrm{D}_{3}$ (notation from $W e b b$ and Fox-Kemper [2011]) to account for spreading.

[80] The preceding scalings account for many of the differences when waves and winds are misaligned, but conditional averaging reveals subtle changes to the dominant two-dimensional LC structures and vorticity budget with increasing misalignment. The averaging reveals that, as the angle between the wind and waves increases, a strong asymmetry is introduced in the vorticity fields, so that in the typical LC vortex pair the upwind cell strengthens and overwhelms a weakened downwind cell. Advection of the upwind cell on top of the downwind cell is the primary forcing of the asymmetric pattern seen in these simulations when $\theta_{w w} \neq 0^{\circ}$. The cross-cell advection under misalignment occurs in both rotating and non-rotating simulations. Interestingly, cross-cell advection in the misaligned, nonrotating simulations resembles cross-cell advection by Ekman processes in the aligned, rotating simulations.

[81] The conclusions of this study are robust. With or without an initial mixed layer, with or without wind waves, and doubling the resolution leave the primary results unchanged. A number of tests could be carried out to increase the realism of these simulations, but we have endeavored to find behaviors likely to be understandable and independent of situation and hence only the cleanest simulations are studied. It is hoped that future studies may address why the production ratio in the TKE budget is time dependent, the case of superposition of two nearly matched wave spectra, and what sets the depth and entrainment of LT under realistic forcing and evolving mixed layer depth.

[82] Acknowledgments. Conversations with Adrean Webb, Greg Chini, Keith Julien, Ramsey Harcourt, Stephen Belcher, and Eric D'Asaro are happily recognized as improving this work. LVR, BFK, and SRH acknowledge the support of NSF 0934737. BFK was also supported by NASA NNX09AF38G. PPS and the computing time for this work were supported by the National Center for Atmospheric Research. PEH was supported by a National Research Council Research Associateship Award at the Naval Research Laboratory and NSF 0934737.

\section{References}

Cavaleri, L., B. Fox-Kemper, and M. Hemer (2012), Wind-waves in the coupled climate system, Bull. Am. Meteorol. Soc., in press.

Cox, S. (1997), Onset of Langmuir circulation when shear flow and Stokes drift are not parallel, Fluid Dyn. Res., 19(3), 149-167.

Craig, P., and M. Banner (1994), Modeling wave-enhanced turbulence in the ocean surface-layer, J. Phys. Oceanogr., 24(12), 2546-2559.

Craik, A. D. D. (1977), Generation of Langmuir circulations by an instability mechanism, J. Fluid Mech., 81, 209-223.

D’Asaro, E., and G. Dairiki (1997), Turbulence intensity measurements in a wind-driven mixed layer, J. Phys. Oceanogr., 27(9), 2009-2022.

de Boyer Montégut, C., G. Madec, A. S. Fischer, A. Lazar, and D. Iudicone (2004), Mixed layer depth over the global ocean: An examination of profile data and a profile-based climatology, J. Geophys. Res., 109, C12003, doi:10.1029/2004JC002378.

Donelan, M., J. Hamilton, and W. Hui (1985), Directional spectra of wind-generated waves, Philos. Trans. R. Soc. London Ser. A, 315(1534), 509-562.

Fox-Kemper, B., R. Ferrari, and R. W. Hallberg (2008), Parameterization of mixed layer eddies. Part I: Theory and diagnosis, J. Phys. Oceanogr., 38(6), 1145-1165, doi:10.1175/2007JPO3792.1.

Fox-Kemper, B., G. Danabasoglu, R. Ferrari, S. Griffies, R. Hallberg, M. Holland, M. Maltrud, S. Peacock, and B. Samuels (2011), Parameterization of mixed-layer eddies. Part III: Implementation and impact in global ocean climate simulations, Ocean Modell., 39, 61-78.

Gnanadesikan, A., and R. Weller (1995), Structure and instability of the Ekman spiral in the presence of surface gravity waves, J. Phys. Oceanogr., 25(12), 3148-3171.

Grant, A. L. M., and S. E. Belcher (2009), Characteristics of Langmuir turbulence in the ocean mixed layer, J. Phys. Oceanogr., 39(8), 1871-1887, doi:10.1175/2009JPO4119.1.

Hanley, K. E., S. E. Belcher, and P. P. Sullivan (2010), A global climatology of wind-wave interaction, J. Phys. Oceanogr., 40(6), 1263-1282, doi:10.1175/2010JPO4377.1.

Hanley, K., S. Belcher, and P. Sullivan (2011), Reply, J. Phys. Oceanogr., 41, 1814-1817, doi:10.1175/JPO-D-11-051.1.

Harcourt, R. R., and E. A. D'Asaro (2008), Large-eddy simulation of Langmuir turbulence in pure wind seas, J. Phys. Oceanogr., 38(7), 1542-1562, doi:10.1175/2007JPO3842.1.

Högström, U., A.-S. Smedman, A. Semedo, and A. Rutgersson (2011), Comments on "A global climatology of windwave interaction," J. Phys. Oceanogr., 41, 1811-1813, doi:10.1175/JPO-D-10-05015.1.

Holm, D. (1996), The ideal Craik-Leibovich equations, Phys. D, 98(2-4), 415-441.

Kukulka, T., A. J. Plueddemann, J. H. Trowbridge, and P. P. Sullivan (2009), Significance of Langmuir circulation in upper ocean mixing: Comparison of observations and simulations, Geophys. Res. Lett., 36 , L10603, doi:10.1029/2009GL037620.

Kukulka, T., A. J. Plueddemann, J. H. Trowbridge, and P. P. Sullivan (2010), Rapid mixed layer deepening by the combination of Langmuir and shear instabilities: A case study, J. Phys. Oceanogr., 40(11), 2381-2400, doi:10.1175/2010JPO4403.1.

Langmuir, I. (1938), Surface motion of water induced by wind, Science, $87,119-123$.

Large, W., G. Danabasoglu, S. Doney, and J. McWilliams (1997), Sensitivity to surface forcing and boundary layer mixing in a global ocean model: Annual-mean climatology, J. Phys. Oceanogr., 27(11), 2418-2447.

Leibovich, S. (1983), The form and dynamics of Langmuir circulations, Annu. Rev. Fluid Mech., 15, 391-427.

Li, M., C. Garrett, and E. Skyllingstad (2005), A regime diagram for classifying turbulent large eddies in the upper ocean, Deep Sea Res. Part I, 52(2), 259-278, doi:10.1016/j.dsr.2004.09.004.

McWilliams, J., and P. Sullivan (2000), Vertical mixing by Langmuir circulations, Spill Sci. Technol. Bull., 6(3-4), 225-237.

McWilliams, J., P. Sullivan, and C. Moeng (1997), Langmuir turbulence in the ocean, J. Fluid Mech., 334, 1-30.

Moeng, C.-H. (1984), A large-eddy-simulation model for the study of planetary boundary-layer turbulence, J. Atmos. Sci., 41, 2052-2062.

Noh, Y., H. Min, and S. Raasch (2004), Large eddy simulation of the ocean mixed layer: The effects of wave breaking and Langmuir circulation, J. Phys. Oceanogr., 34(4), 720-735.

Polonichko, V. (1997), Generation of Langmuir circulation for nonaligned wind stress and the Stokes drift, J. Geophys. Res., 102(C7), 15,773-15,780.

Polton, J. A., and S. E. Belcher (2007), Langmuir turbulence and deeply penetrating jets in an unstratified mixed layer, J. Geophys. Res., 112, C09020, doi:10.1029/2007JC004205.

Skyllingstad, E., and D. Denbo (1995), An ocean large-eddy simulation of Langmuir circulations and convection in the surface mixed-layer, J. Geophys. Res., 100(C5), 8501-8522.

Smith, J. (1992), Observed growth of Langmuir circulation, J. Geophys. Res., 97(C4), 5651-5664.

Sullivan, P. P., and J. C. McWilliams (2010), Dynamics of winds and currents coupled to surface waves, Annu. Rev. Fluid Mech., 42, 19-42, doi:10.1146/annurev-fluid-121108-145541.

Sullivan, P., J. McWilliams, and C. Moeng (1996), A grid nesting method for large-eddy simulation of planetary boundary-layer flows, Boundary Layer Meteorol., 80(1-2), 167-202.

Sullivan, P., J. McWilliams, and W. Melville (2004), The oceanic boundary layer driven by wave breaking with stochastic variability. Part 1 . Direct numerical simulations, J. Fluid Mech., 507, 143-174.

Sullivan, P. P., J. C. Mcwilliams, and W. K. Melville (2007), Surface gravity wave effects in the oceanic boundary layer: Large-eddy simulation with 
vortex force and stochastic breakers, J. Fluid Mech., 593, 405-452, doi: $10.1017 / \mathrm{S} 002211200700897 \mathrm{X}$

Thorpe, S. A. (2007), An Introduction to Ocean Turbulence, Cambridge Univ. Press, Cambridge, U. K.

Webb, A., and B. Fox-Kemper (2011), Wave spectral moments and Stokes drift estimation, Ocean Modell., 40(3-4), 273-288, doi:10.1016/ j.ocemod.2011.08.007.

Weller, R., and J. Price (1988), Langmuir circulation within the oceanic mixed layer, Deep Sea Res. Part A, 35(5), 711-747.

B. Fox-Kemper and L. P. Van Roekel, Cooperative Institute for Research in Environmental Sciences, University of Colorado Boulder, UCB 216,
Boulder, CO 80309, USA. (bfk@colorado.edu; luke.vanroekel@colorado. edu)

P. E. Hamlington, Aerospace Engineering Sciences, University of Colorado Boulder, UCB 429, Boulder, CO 80309, USA. (peter hamlington@colorado.edu)

S. R. Haney, Department of Atmospheric and Oceanic Science, University of Colorado Boulder, UCB 216, Boulder, CO 80309, USA (sean.r.haney@colorado.edu)

P. P. Sullivan, Mesoscale and Microscale Meteorology Division, National Center for Atmospheric Research, PO Box 3000, Boulder, CO 80307, USA (pps@ucar.edu) 